\title{
Existence and uniqueness of positive solutions for singular fractional differential systems with coupled integral boundary conditions
}

\author{
Lishan Liü,b,*, Hongdan Lia ${ }^{a}$ Chun Liü, Yonghong Wu \\ a School of Mathematical Sciences, Qufu Normal University, 273165, Qufu, China. \\ ${ }^{b}$ Department of Mathematics and Statistics, Curtin University, WA6845, Perth, Australia. \\ Communicated by Y. H. Yao
}

\begin{abstract}
In this paper, we study the existence and uniqueness of positive solutions for a class of singular fractional differential systems with coupled integral boundary conditions. By using the properties of the Green function, the mixed monotone method and the fixed point theory in cones, we obtain the existence and uniqueness results for the problem. The results obtained herein generalize and improve some known results including singular and non-singular cases. (C)2017 All rights reserved.
\end{abstract}

Keywords: Singular fractional differential equations, Riemann-Stieltjes integral boundary value problem, positive solution, fixed point theorem in cone.

2010 MSC: 34B16, 34B18, 47H05.

\section{Introduction}

In this article, we consider the existence and uniqueness of positive solutions for a class of singular fractional differential systems with coupled integral boundary conditions as follows

$$
\left\{\begin{array}{l}
D_{0^{+}}^{\alpha} u(t)+p_{1}(t) f_{1}(t, u(t), v(t))+q_{1}(t) g_{1}(t, u(t), v(t))=0, \quad t \in(0,1), \\
D_{0^{+}}^{\beta} v(t)+p_{2}(t) f_{2}(t, u(t), v(t))+q_{2}(t) g_{2}(t, u(t), v(t))=0, \quad t \in(0,1), \\
u(0)=u^{\prime}(0)=\cdots=u^{(n-2)}(0)=0, \quad u(1)=\int_{0}^{1} a(s) v(s) d A(s), \\
v(0)=v^{\prime}(0)=\cdots=v^{(m-2)}(0)=0, \quad v(1)=\int_{0}^{1} b(s) u(s) d B(s)
\end{array}\right.
$$

where $\alpha, \beta \in \mathbb{R}, n-1<\alpha \leqslant n, m-1<\beta \leqslant m, n, m \in \mathbb{N}, n, m \geqslant 2, D_{0^{+}}^{\alpha}$ and $D_{0^{+}}^{\beta}$ denote the RiemannLiouville derivatives of orders $\alpha$ and $\beta$, respectively. $p_{i}, q_{i} \in C((0,1),[0, \infty)), a, b \in C([0,1],[0, \infty))$,

\footnotetext{
*Corresponding author

Email addresses: mathlls@163.com (Lishan Liu), Ihd200908@163.com (Hongdan Li), liuchunjn@aliyun.com (Chun Liu), yhwu@maths . curtin. edu.au (Yonghong $\mathrm{Wu}$ )
}

doi:10.22436/jnsa.010.01.24 
$f_{i} \in C((0,1) \times(0, \infty) \times(0, \infty),[0, \infty)), g_{i} \in C((0,1) \times(0, \infty) \times(0, \infty),[0, \infty))$ and $f_{i}(t, x, y)$ may be singular at $t=0,1$ and $y=0$, and $g_{i}(t, x, y)$ may be singular at $t=0,1$ and $x=0(i=1,2) . \int_{0}^{1} a(s) v(s) d A(s)$, $\int_{0}^{1} b(s) u(s) d B(s)$ denote the Riemann-Stieltjes integral with a signed measure, that is, $A, B:[0,1] \rightarrow[0, \infty)$ are functions of boundary variation. By a positive solution of BVP (1.1), we mean a pair of functions $(u, v) \in \mathrm{C}[0,1] \times \mathrm{C}[0,1]$ satisfying BVP $(1.1)$ with $u(t)>0$ and $v(t)>0$ for all $t \in(0,1]$.

In recent years, boundary value problems for a coupled system of nonlinear differential equations have gained its popularity and importance due to its various applications in heat conduction, chemical engineering, underground water flow, thermo-elasticity and plasma physics. There have appeared some results for the existence of solutions or positive solutions of boundary value problems for a coupled system of nonlinear fractional differential equations, see $[1,2,4,6,7,9,11-13,16,18-23,25]$ and the references therein. Most of the results show that the equations have either single or multiple positive solutions.

In [3], Cui et al. investigated the following singular problem

$$
\left\{\begin{array}{l}
-x^{\prime \prime}(t)=f(t, x(t), y(t)), \quad t \in(0,1) \\
-y^{\prime \prime}(t)=g(t, x(t), y(t)), \quad t \in(0,1) \\
x(0)=\int_{0}^{1} y(t) d \alpha(t), \quad y(0)=\int_{0}^{1} x(t) d \beta(t), \\
x(1)=y(1)=0,
\end{array}\right.
$$

where $\int_{0}^{1} y(t) d \alpha(t)$ and $\int_{0}^{1} x(t) d \beta(t)$ denote the Riemann-Stieltjes integrals of $y$ and $x$ with respect to $\alpha$ and $\beta$, respectively; $f \in C((0,1) \times[0, \infty) \times(0, \infty),[0, \infty)), g \in C((0,1) \times(0, \infty) \times[0, \infty),[0, \infty))$ and $f(t, x, y)$ is nondecreasing in $x$ and nonincreasing in $y$ and may be singular at $t=0,1$ and $y=0$, while $g(t, x, y)$ is nonincreasing in $x$ and nondecreasing in $y$ and may be singular at $t=0,1$ and $x=0$.

In [21], Wang et al. considered the following singular fractional differential system with coupled boundary conditions

$$
\left\{\begin{array}{l}
D_{0^{+}}^{\alpha_{1}} u(t)+f(t, u(t), v(t))=0, \\
D_{0^{+}}^{\alpha_{2}} v(t)+g(t, u(t), v(t))=0, \quad t \in(0,1), \\
u(0)=u^{\prime}(0)=\cdots=u^{(n-2)}=0, \quad u(1)=\mu_{1} \int_{0}^{1} v(s) d A_{1}(s), \\
v(0)=v^{\prime}(0)=\cdots=v^{(n-2)}=0, \quad v(1)=\mu_{2} \int_{0}^{1} u(s) d A_{2}(s),
\end{array}\right.
$$

where $\mathrm{n}-1<\alpha_{\mathrm{i}} \leqslant \mathrm{n}, \mathrm{n} \geqslant 2$, and $\mathrm{D}_{0^{+}}^{\alpha_{i}}$ is the standard Riemann-Liouvill derivative. $\mathrm{f} \in \mathrm{C}((0,1) \times[0, \infty) \times$ $(0, \infty),[0, \infty)), \mathrm{g} \in \mathrm{C}((0,1) \times(0, \infty) \times[0, \infty),[0, \infty))$ and $f(t, x, y)$ is nondecreasing in $x$ and nonincreasing in $y$ and may be singular at $t=0,1$ and $y=0$, while $g(t, x, y)$ is nonincreasing in $x$ and nondecreasing in $y$ and may be singular at $t=0,1$ and $x=0$. By using the Guo-Krasnosel'skii fixed point theorem, they obtained the existence of a positive solution and the uniqueness of the positive solution under the condition $\alpha_{1}=\alpha_{2}$.

In [8], Henderson and Luca studied the system of nonlinear fractional differential equations

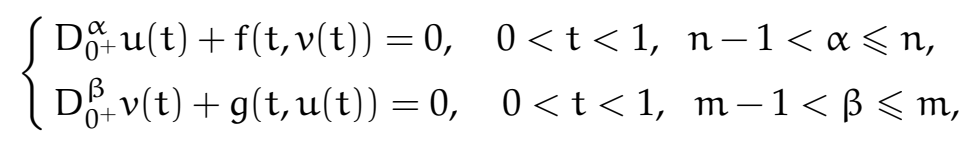

with the integral boundary conditions

$$
\begin{cases}u(0)=u^{\prime}(0)=\cdots=u^{(n-2)}(0)=0, & u(1)=\int_{0}^{1} u(s) d H(s), \\ v(0)=v^{\prime}(0)=\cdots=v^{(m-2)}(0)=0, & v(1)=\int_{0}^{1} v(s) d K(s),\end{cases}
$$


where $n, m \in \mathbb{N}, n, m \geqslant 2, D_{0^{+}}^{\alpha}$ and $D_{0^{+}}^{\beta}$ denote the Riemann-Liouville derivatives of orders $\alpha$ and $\beta$ respectively and $f, g:[0,1] \times[0, \infty) \rightarrow[0, \infty)$ are continuous and $f(t, 0)=g(t, 0)=0$ for all $t \in[0,1]$. They obtained the existence and multiplicity of positive solutions for the above BVP by using the GuoKrasnosel'skii fixed point theorem and some theorems from the fixed point index theory, but they did not discuss the uniqueness of positive solutions.

Motivated by the above mentioned work, the purpose of this article is to investigate the existence and uniqueness of positive solutions for singular fractional differential systems with coupled integral boundary conditions under certain conditions on the functions $f_{i}$ and $g_{i}(i=1,2)$. The main new features presented in this paper are as follows. Firstly, we divided the functions of the BVP into $f_{i}$ and $g_{i}$ so that the boundary value problem has a more general form. Secondly, $D_{0^{+}}^{\alpha}$ and $D_{0^{+}}^{\beta}$ denote the Riemann-Liouville derivatives of orders $\alpha$ and $\beta$ in which $\alpha \in(n-1, n], \beta \in(m-1, m], n, m \in \mathbb{N}$. Thirdly, if $d A(s)=d B(s)=d s$ or $h(s) d s$, then BVP (1.1) reduces to a multi-point boundary value problem as a special case. Fourthly, the nonlinearity is allowed to be singular in regard to time and space variable elements. In particular, for any $\alpha, \beta \in(0,+\infty)$, we obtain the existence and uniqueness of positive solutions for singular fractional differential systems (1.1). The results obtained herein generalize and improve some known results including singular and non-singular cases.

The rest of the paper is organized as follows. In Section 2, we present the necessary definitions and properties to prove our main results, and obtain the corresponding Green function and some of its properties. In Section 3, we give the existence and uniqueness theorem for the positive solutions with respect to a cone for the BVP (1.1). In Section 4, as an application, an interesting example is presented to illustrate the main result. Conclusions are presented in Section 5.

\section{Preliminaries and lemmas}

For the convenience of the reader, we present some definitions and lemmas to be used in the proof of our main result. They also can be found in the literature $[10,14,15,17]$.

Definition 2.1. The Riemann-Liouville fractional integral of order $\alpha>0$ of a function $y:(0, \infty) \rightarrow \mathbb{R}$ is given by

$$
\mathrm{I}_{0+}^{\alpha} \mathrm{y}(\mathrm{t})=\frac{1}{\Gamma(\alpha)} \int_{0}^{\mathrm{t}}(\mathrm{t}-\mathrm{s})^{\alpha-1} \mathrm{y}(\mathrm{s}) \mathrm{ds},
$$

provided that the right-hand side is pointwise defined on $(0, \infty)$.

Definition 2.2. The Riemann-Liouville fractional derivative of order $\alpha>0$ of a continuous function $y:(0, \infty) \rightarrow \mathbb{R}$ is given by

$$
D_{0+}^{\alpha} y(t)=\frac{1}{\Gamma(n-\alpha)}\left(\frac{d}{d t}\right)^{n} \int_{0}^{t} \frac{y(s)}{(t-s)^{\alpha-n+1}} d s
$$

where $n=[\alpha]+1,[\alpha]$ denotes the integer part of the number $\alpha$, provided that the right hand side is pointwise defined on $(0, \infty)$.

Lemma 2.3 ([10]). Let $\alpha>0$. If we assume $\mathrm{u} \in \mathrm{C}(0,1) \cap \mathrm{L}(0,1)$, then the fractional differential equation

$$
\mathrm{D}_{0+}^{\alpha} \mathrm{u}(\mathrm{t})=0 \text {, }
$$

has $\mathrm{u}(\mathrm{t})=\mathrm{C}_{1} \mathrm{t}^{\alpha-1}+\mathrm{C}_{2} \mathrm{t}^{\alpha-2}+\cdots+\mathrm{C}_{\mathrm{N}} \mathrm{t}^{\alpha-\mathrm{N}}, \mathrm{C}_{\mathrm{i}} \in \mathbb{R}(\mathrm{i}=1,2, \cdots, \mathrm{N})$ as the unique solution, where $\mathrm{N}=$ $[\alpha]+1$.

From the definition of the Riemann-Liouville derivative, we can obtain the statement.

Lemma 2.4 ([10]). Assume that $u \in C(0,1) \cap \mathrm{L}(0,1)$ with a fractional derivative of order $\alpha>0$ that belongs to $\mathrm{C}(0,1) \cap \mathrm{L}(0,1)$. Then

$$
\mathrm{I}_{0+}^{\alpha} \mathrm{D}_{0+}^{\alpha} \mathrm{u}(\mathrm{t})=\mathrm{u}(\mathrm{t})+\mathrm{C}_{1} \mathrm{t}^{\alpha-1}+\mathrm{C}_{2} \mathrm{t}^{\alpha-2}+\cdots+\mathrm{C}_{\mathrm{N}} \mathrm{t}^{\alpha-\mathrm{N}}
$$

for some $\mathrm{C}_{i} \in \mathbb{R}(i=1,2, \cdots, \mathrm{N})$, where $\mathrm{N}=[\alpha]+1$. 
In the following, we present the Green function of the fractional differential equation boundary value problem.

Lemma 2.5. Let $x, y \in C(0,1) \cap \mathrm{L}^{1}(0,1)$ be given functions. Then the boundary value problem

$$
\left\{\begin{array}{l}
D_{0+}^{\alpha} u(t)+x(t)=0, \quad 0<t<1, \quad n-1<\alpha \leqslant n \\
D_{0+}^{\beta} v(t)+y(t)=0, \quad 0<t<1, \quad m-1<\beta \leqslant m \\
u(0)=u^{\prime}(0)=\cdots=u^{(n-2)}(0)=0, \quad u(1)=\int_{0}^{1} a(s) v(s) d A(s), \\
v(0)=v^{\prime}(0)=\cdots=v^{(m-2)}(0)=0, \quad v(1)=\int_{0}^{1} b(s) u(s) d B(s),
\end{array}\right.
$$

where $\mathrm{n}, \mathrm{m} \in \mathbb{N}, \mathrm{n}, \mathrm{m} \geqslant 2$, is equivalent to

$$
\begin{cases}u(t)=\int_{0}^{1} G_{1}(t, s) x(s) d s+\int_{0}^{1} H_{1}(t, s) y(s) d s, & t \in[0,1], \\ v(t)=\int_{0}^{1} G_{2}(t, s) y(s) d s+\int_{0}^{1} H_{2}(t, s) x(s) d s, & t \in[0,1]\end{cases}
$$

where

$$
\left\{\begin{array}{l}
\mathrm{G}_{1}(\mathrm{t}, \mathrm{s})=\mathrm{g}_{1}(\mathrm{t}, \mathrm{s})+\frac{\Delta_{1}}{\Delta} \mathrm{t}^{\alpha-1} \int_{0}^{1} g_{1}(\tau, \mathrm{s}) \mathrm{b}(\tau) \mathrm{dB}(\tau), \\
\mathrm{H}_{1}(\mathrm{t}, \mathrm{s})=\frac{\mathrm{t}^{\alpha-1}}{\Delta} \int_{0}^{1} g_{2}(\tau, \mathrm{s}) \mathrm{a}(\tau) \mathrm{dA}(\tau), \\
\mathrm{G}_{2}(\mathrm{t}, \mathrm{s})=\mathrm{g}_{2}(\mathrm{t}, \mathrm{s})+\frac{\Delta_{2}}{\Delta} \mathrm{t}^{\beta-1} \int_{0}^{1} g_{2}(\tau, s) a(\tau) d A(\tau), \\
\mathrm{H}_{2}(\mathrm{t}, \mathrm{s})=\frac{\mathrm{t}^{\beta-1}}{\Delta} \int_{0}^{1} g_{1}(\tau, s) b(\tau) d B(\tau),
\end{array}\right.
$$

and

$$
\begin{aligned}
& g_{1}(t, s)=\frac{1}{\Gamma(\alpha)}\left\{\begin{array}{l}
{[t(1-s)]^{\alpha-1}-(t-s)^{\alpha-1}, 0 \leqslant s \leqslant t \leqslant 1,} \\
{[t(1-s)]^{\alpha-1}, \quad 0 \leqslant t \leqslant s \leqslant 1,}
\end{array}\right. \\
& g_{2}(t, s)=\frac{1}{\Gamma(\beta)}\left\{\begin{array}{l}
{[t(1-s)]^{\beta-1}-(t-s)^{\beta-1}, 0 \leqslant s \leqslant t \leqslant 1,} \\
{[t(1-s)]^{\beta-1}, \quad 0 \leqslant t \leqslant s \leqslant 1,}
\end{array}\right.
\end{aligned}
$$

in which $\Delta=1-\Delta_{1} \Delta_{2} \neq 0$, and $\Delta_{1}=\int_{0}^{1} \mathrm{a}(\mathrm{s}) \mathrm{s}^{\beta-1} \mathrm{dA}(\mathrm{s}), \Delta_{2}=\int_{0}^{1} \mathrm{~b}(\mathrm{~s}) \mathrm{s}^{\alpha-1} \mathrm{~dB}(\mathrm{~s})$.

Proof. By Lemmas 2.3 and 2.4, the solution of the system (2.1) is

$$
\left\{\begin{array}{l}
u(t)=-\frac{1}{\Gamma(\alpha)} \int_{0}^{t}(t-s)^{\alpha-1} x(s) d s+c_{1} t^{\alpha-1}+\cdots+c_{n} t^{\alpha-n}, t \in[0,1], \\
v(t)=-\frac{1}{\Gamma(\beta)} \int_{0}^{t}(t-s)^{\beta-1} y(s) d s+d_{1} t^{\beta-1}+\cdots+d_{m} t^{\beta-m}, t \in[0,1],
\end{array}\right.
$$

where $c_{i}, d_{j} \in \mathbb{R}(i=1,2,3, \cdots, n ; j=1,2,3, \cdots, m)$. By using the conditions $u(0)=u^{\prime}(0)=\cdots=$ $u^{(n-2)}(0)=0$ and $v(0)=v^{\prime}(0)=\cdots=v^{(m-2)}(0)=0$, we obtain $c_{2}=c_{3}=\cdots=c_{n}=0$ and $d_{2}=d_{3}=$ $\cdots=d_{m}=0$. Then by (2.6) we conclude

$$
\left\{\begin{array}{l}
u(t)=c_{1} t^{\alpha-1}-\frac{1}{\Gamma(\alpha)} \int_{0}^{t}(t-s)^{\alpha-1} x(s) d s, t \in[0,1] \\
v(t)=d_{1} t^{\beta-1}-\frac{1}{\Gamma(\beta)} \int_{0}^{t}(t-s)^{\beta-1} y(s) d s, t \in[0,1]
\end{array}\right.
$$


Combining (2.7) with the conditions $u(1)=\int_{0}^{1} a(s) v(s) d A(s)$ and $v(1)=\int_{0}^{1} b(s) u(s) d B(s)$, we deduce

$$
\left\{\begin{array}{l}
c_{1}-\frac{1}{\Gamma(\alpha)} \int_{0}^{1}(1-s)^{\alpha-1} \chi(s) d s=\int_{0}^{1} a(s)\left[d_{1} s^{\beta-1}-\frac{1}{\Gamma(\beta)} \int_{0}^{s}(s-\tau)^{\beta-1} y(\tau) d \tau\right] d A(s), \\
d_{1}-\frac{1}{\Gamma(\beta)} \int_{0}^{1}(1-s)^{\beta-1} y(s) d s=\int_{0}^{1} b(s)\left[c_{1} s^{\alpha-1}-\frac{1}{\Gamma(\alpha)} \int_{0}^{s}(s-\tau)^{\alpha-1} \chi(\tau) d \tau\right] d B(s),
\end{array}\right.
$$

or equivalently

$$
\left\{\begin{array}{l}
c_{1}-d_{1} \int_{0}^{1} a(s) s^{\beta-1} d A(s)=\frac{1}{\Gamma(\alpha)} \int_{0}^{1}(1-s)^{\alpha-1} \chi(s) d s-\frac{1}{\Gamma(\beta)} \int_{0}^{1} \int_{s}^{1} a(\tau)(\tau-s)^{\beta-1} d A(\tau) y(s) d s, \\
d_{1}-c_{1} \int_{0}^{1} b(s) s^{\alpha-1} d B(s)=\frac{1}{\Gamma(\beta)} \int_{0}^{1}(1-s)^{\beta-1} y(s) d s-\frac{1}{\Gamma(\alpha)} \int_{0}^{1} \int_{s}^{1} b(\tau)(\tau-s)^{\alpha-1} d B(\tau) \chi(s) d s .
\end{array}\right.
$$

The above system in the unknowns $c_{1}$ and $d_{1}$ has the determinant

$$
\begin{aligned}
\Delta & =\left|\begin{array}{cc}
1 & -\int_{0}^{1} a(s) s^{\beta-1} d A(s) \\
-\int_{0}^{1} b(s) s^{\alpha-1} d B(s) & 1
\end{array}\right| \\
& =1-\left(\int_{0}^{1} a(s) s^{\beta-1} d A(s)\right)\left(\int_{0}^{1} b(s) s^{\alpha-1} d B(s)\right) \\
& =1-\Delta_{1} \Delta_{2} .
\end{aligned}
$$

So by (2.8) and (2.9) we obtain

$$
\begin{aligned}
c_{1}= & \frac{1}{\Delta}\left[\frac{1}{\Gamma(\alpha)} \int_{0}^{1}(1-s)^{\alpha-1} \chi(s) d s-\frac{\Delta_{1}}{\Gamma(\alpha)} \int_{0}^{1} \int_{s}^{1} b(\tau)(\tau-s)^{\alpha-1} \mathrm{~dB}(\tau) \chi(s) d s\right. \\
& \left.-\frac{1}{\Gamma(\beta)} \int_{0}^{1} \int_{s}^{1} \mathrm{a}(\tau)(\tau-s)^{\beta-1} \mathrm{dA}(\tau) y(s) d s+\frac{\Delta_{1}}{\Gamma(\beta)} \int_{0}^{1}(1-s)^{\beta-1} y(s) d s\right] \\
\mathrm{d}_{1}= & \frac{1}{\Delta}\left[\frac{1}{\Gamma(\beta)} \int_{0}^{1}(1-s)^{\beta-1} y(s) d s-\frac{\Delta_{2}}{\Gamma(\beta)} \int_{0}^{1} \int_{s}^{1} a(\tau)(\tau-s)^{\beta-1} \mathrm{dA}(\tau) y(s) d s\right. \\
& \left.-\frac{1}{\Gamma(\alpha)} \int_{0}^{1} \int_{s}^{1} b(\tau)(\tau-s)^{\alpha-1} \mathrm{~dB}(\tau) x(s) d s+\frac{\Delta_{2}}{\Gamma(\alpha)} \int_{0}^{1}(1-s)^{\alpha-1} x(s) d s\right]
\end{aligned}
$$

Therefore, by combining (2.7) with (2.10) and (2.11), we deduce

$$
\begin{aligned}
u(t)= & -\frac{1}{\Gamma(\alpha)} \int_{0}^{t}(t-s)^{\alpha-1} \chi(s) d s+\frac{t^{\alpha-1}}{\Delta}\left[\frac{1}{\Gamma(\alpha)} \int_{0}^{1}(1-s)^{\alpha-1} \chi(s) d s\right. \\
& -\frac{\Delta_{1}}{\Gamma(\alpha)} \int_{0}^{1} \int_{s}^{1} b(\tau)(\tau-s)^{\alpha-1} d B(\tau) x(s) d s \\
& \left.-\frac{1}{\Gamma(\beta)} \int_{0}^{1} \int_{s}^{1} a(\tau)(\tau-s)^{\beta-1} d A(\tau) y(s) d s+\frac{\Delta_{1}}{\Gamma(\beta)} \int_{0}^{1}(1-s)^{\beta-1} y(s) d s\right], \\
v(t)= & -\frac{1}{\Gamma(\beta)} \int_{0}^{t}(t-s)^{\beta-1} y(s) d s+\frac{t^{\beta-1}}{\Delta}\left[\frac{1}{\Gamma(\beta)} \int_{0}^{1}(1-s)^{\beta-1} y(s) d s\right. \\
& -\frac{\Delta_{2}}{\Gamma(\beta)} \int_{0}^{1} \int_{s}^{1} a(\tau)(\tau-s)^{\beta-1} d A(\tau) y(s) d s \\
& \left.-\frac{1}{\Gamma(\alpha)} \int_{0}^{1} \int_{s}^{1} b(\tau)(\tau-s)^{\alpha-1} d B(\tau) x(s) d s+\frac{\Delta_{2}}{\Gamma(\alpha)} \int_{0}^{1}(1-s)^{\alpha-1} \chi(s) d s\right] .
\end{aligned}
$$


We conclude

$$
\begin{aligned}
& u(t)=\frac{1}{\Gamma(\alpha)}\left[\int_{0}^{t}\left[t^{\alpha-1}(1-s)^{\alpha-1}-(t-s)^{\alpha-1}\right] x(s) d s\right. \\
& \left.+\int_{t}^{1} t^{\alpha-1}(1-s)^{\alpha-1} \chi(s) d s-\int_{0}^{1} t^{\alpha-1}(1-s)^{\alpha-1} \chi(s) d s\right] \\
& +\frac{t^{\alpha-1}}{\Delta \Gamma(\alpha)} \int_{0}^{1}(1-s)^{\alpha-1} \chi(s) d s-\frac{\Delta_{1}}{\Delta \Gamma(\alpha)} t^{\alpha-1} \int_{0}^{1} \int_{s}^{1} b(\tau)(\tau-s)^{\alpha-1} d B(\tau) x(s) d s \\
& +\frac{t^{\alpha-1}}{\Delta \Gamma(\beta)}\left[\int_{0}^{1} \int_{0}^{1} a(\tau) \tau^{\beta-1}(1-s)^{\beta-1} d A(\tau) y(s) d s\right. \\
& \left.-\int_{0}^{1} \int_{s}^{1} a(\tau)(\tau-s)^{\beta-1} d A(\tau) y(s) d s\right] \\
& =\frac{1}{\Gamma(\alpha)}\left[\int_{0}^{t}\left[t^{\alpha-1}(1-s)^{\alpha-1}-(t-s)^{\alpha-1}\right] x(s) d s\right. \\
& +\int_{t}^{1} t^{\alpha-1}(1-s)^{\alpha-1} \chi(s) d s-\frac{1}{\Delta} \int_{0}^{1} t^{\alpha-1}(1-s)^{\alpha-1} \chi(s) d s \\
& +\frac{\Delta_{1} \Delta_{2}}{\Delta} \int_{0}^{1} \mathrm{t}^{\alpha-1}(1-\mathrm{s})^{\alpha-1} \chi(\mathrm{s}) \mathrm{ds}+\frac{1}{\Delta} \int_{0}^{1} \mathrm{t}^{\alpha-1}(1-\mathrm{s})^{\alpha-1} \chi(\mathrm{s}) \mathrm{d} s \\
& \left.-\frac{\Delta_{1}}{\Delta} \mathrm{t}^{\alpha-1} \int_{0}^{1} \int_{s}^{1} \mathrm{~b}(\tau)(\tau-\mathrm{s})^{\alpha-1} \mathrm{~dB}(\tau) x(\mathrm{~s}) \mathrm{ds}\right] \\
& +\frac{t^{\alpha-1}}{\Delta \Gamma(\beta)}\left[\int_{0}^{1} \int_{0}^{1} a(\tau) \tau^{\beta-1}(1-s)^{\beta-1} d A(\tau) y(s) d s-\int_{0}^{1} \int_{s}^{1} a(\tau)(\tau-s)^{\beta-1} d A(\tau) y(s) d s\right] \\
& =\frac{1}{\Gamma(\alpha)}\left\{\int_{0}^{t}\left[t^{\alpha-1}(1-s)^{\alpha-1}-(t-s)^{\alpha-1}\right] x(s) d s+\int_{t}^{1} t^{\alpha-1}(1-s)^{\alpha-1} \chi(s) d s+\frac{\Delta_{1}}{\Delta} t^{\alpha-1}\right. \\
& \left.\times\left[\int_{0}^{1} \int_{0}^{1} \mathrm{~b}(\tau)(\tau)^{\alpha-1}(1-s)^{\alpha-1} \mathrm{~dB}(\tau) x(s) \mathrm{d} s-\int_{0}^{1} \int_{s}^{1} \mathrm{~b}(\tau)(\tau-s)^{\alpha-1} \mathrm{~dB}(\tau) x(s) \mathrm{d} s\right]\right\} \\
& +\frac{t^{\alpha-1}}{\Delta \Gamma(\beta)}\left[\int_{0}^{1} \int_{0}^{1} a(\tau) \tau^{\beta-1}(1-s)^{\beta-1} d A(\tau) y(s) d s-\int_{0}^{1} \int_{s}^{1} a(\tau)(\tau-s)^{\beta-1} d A(\tau) y(s) d s\right] .
\end{aligned}
$$

Therefore, we obtain

$$
\begin{aligned}
u(t)= & \frac{1}{\Gamma(\alpha)}\left\{\int_{0}^{t}\left[t^{\alpha-1}(1-s)^{\alpha-1}-(t-s)^{\alpha-1}\right] x(s) d s+\int_{t}^{1} t^{\alpha-1}(1-s)^{\alpha-1} x(s) d s+\frac{\Delta_{1}}{\Delta} t^{\alpha-1}\right. \\
& \times\left[\int_{0}^{1} \int_{0}^{s} b(\tau)(\tau)^{\alpha-1}(1-s)^{\alpha-1} d B(\tau) x(s) d s+\int_{0}^{1} \int_{s}^{1} b(\tau)(\tau)^{\alpha-1}(1-s)^{\alpha-1} d B(\tau) x(s) d s\right. \\
& \left.\left.-\int_{0}^{1} \int_{s}^{1} b(\tau)(\tau-s)^{\alpha-1} d B(\tau) x(s) d s\right]\right\} \\
& +\frac{t^{\alpha-1}}{\Delta \Gamma(\beta)}\left[\int_{0}^{1} \int_{0}^{s} a(\tau) \tau^{\beta-1}(1-s)^{\beta-1} d A(\tau) y(s) d s\right. \\
& \left.+\int_{s}^{1} \int_{0}^{1} a(\tau) \tau^{\beta-1}(1-s)^{\beta-1} d A(\tau) y(s) d s-\int_{0}^{1} \int_{s}^{1} a(\tau)(\tau-s)^{\beta-1} d A(\tau) y(s) d s\right]
\end{aligned}
$$




$$
\begin{aligned}
= & \frac{1}{\Gamma(\alpha)}\left\{\int_{0}^{t}\left[t^{\alpha-1}(1-s)^{\alpha-1}-(t-s)^{\alpha-1}\right] x(s) d s+\int_{t}^{1} t^{\alpha-1}(1-s)^{\alpha-1} x(s) d s\right. \\
& +\frac{\Delta_{1}}{\Delta} t^{\alpha-1}\left[\int_{0}^{1} \int_{0}^{s} b(\tau)(\tau)^{\alpha-1}(1-s)^{\alpha-1} d B(\tau) x(s) d s\right. \\
& \left.\left.+\int_{0}^{1} \int_{s}^{1} b(\tau)\left[\tau^{\alpha-1}(1-s)^{\alpha-1}-(\tau-s)^{\alpha-1}\right] d B(\tau) x(s) d s\right]\right\} \\
& +\frac{t^{\alpha-1}}{\Delta \Gamma(\beta)}\left[\int_{0}^{1} \int_{0}^{s} a(\tau) \tau^{\beta-1}(1-s)^{\beta-1} d A(\tau) y(s) d s\right. \\
& \left.+\int_{0}^{1} \int_{s}^{1} a(\tau)\left[\tau^{\beta-1}(1-s)^{\beta-1}-(\tau-s)^{\beta-1}\right] d A(\tau) y(s) d s\right] \\
= & \int_{0}^{1} g_{1}(t, s) x(s) d s+\frac{\Delta_{1}}{\Delta} t^{\alpha-1} \int_{0}^{1} \int_{0}^{1} g_{1}(\tau, s) b(\tau) d B(\tau) x(s) d s \\
& +\frac{t^{\alpha-1}}{\Delta} \int_{0}^{1} \int_{0}^{1} g_{2}(\tau, s) a(\tau) d A(\tau) y(s) d s \\
= & \int_{0}^{1} G_{1}(t, s) x(s) d s+\int_{0}^{1} H_{1}(t, s) y(s) d s .
\end{aligned}
$$

In a similar manner, we deduce

$$
\begin{aligned}
v(t)= & \int_{0}^{1} g_{2}(t, s) y(s) d s+\frac{\Delta_{2}}{\Delta} t^{\beta-1} \int_{0}^{1} \int_{0}^{1} g_{2}(\tau, s) a(\tau) d A(\tau) y(s) d s \\
& +\frac{t^{\beta-1}}{\Delta} \int_{0}^{1} \int_{0}^{1} g_{1}(\tau, s) b(\tau) d B(\tau) x(s) d s \\
= & \int_{0}^{1} G_{2}(t, s) y(s) d s+\int_{0}^{1} H_{2}(t, s) x(s) d s .
\end{aligned}
$$

Therefore, we obtain the expression (2.2) for the solution of problem (2.1).

Lemma 2.6 ([24]). The functions $g_{1}$ and $g_{2}$ given by (2.4) and (2.5) have the following properties:

$$
\begin{array}{ll}
\frac{t^{\alpha-1}(1-t) s(1-s)^{\alpha-1}}{\Gamma(\alpha)} \leqslant g_{1}(t, s) \leqslant \frac{s(1-s)^{\alpha-1}}{\Gamma(\alpha-1)}\left(\text { or } \frac{t^{\alpha-1}(1-t)^{\alpha-1}}{\Gamma(\alpha)}\right) \quad \forall t, s \in[0,1] \\
\frac{t^{\beta-1}(1-t) s(1-s)^{\beta-1}}{\Gamma(\beta)} \leqslant g_{2}(t, s) \leqslant \frac{s(1-s)^{\beta-1}}{\Gamma(\beta-1)}\left(\text { or } \frac{t^{\beta-1}(1-t)^{\beta-1}}{\Gamma(\beta)}\right), \quad \forall t, s \in[0,1] .
\end{array}
$$

The following properties of the Green function play an important role in this paper.

Lemma 2.7. The Green functions $\mathrm{G}_{i}(\mathrm{t}, \mathrm{s}), \mathrm{H}_{i}(\mathrm{t}, \mathrm{s})(\mathrm{i}=1,2)$ defined by (2.3) have the following properties:

(1) $G_{i}(t, s), H_{i}(t, s)$ are continuous functions on $[0,1] \times[0,1]$ and $G_{i}(t, s), H_{i}(t, s) \geqslant 0, s, t \in[0,1](i=1,2)$;

(2) $G_{i}(t, s) \leqslant k_{1} s(1-s)^{\gamma_{1}} \quad\left(\right.$ or $\left.k_{1} t^{\gamma_{1}}\right), H_{i}(t, s) \leqslant k_{1} s(1-s)^{\gamma_{1}} \quad$ (or $\left.k_{1} t^{\gamma_{1}}\right), G_{i}(t, s) \geqslant k_{2} t^{\gamma_{2}} s(1-$ $s)^{\gamma_{2}}, H_{i}(t, s) \geqslant k_{2} t^{\gamma_{2}} s(1-s)^{\gamma_{2}}(i=1,2)$, where

$$
k_{1}=\max \left\{\frac{\Delta_{1}}{\Delta \Gamma(\alpha-1)} \int_{0}^{1} b(\tau) d B(\tau)+\frac{1}{\Gamma(\alpha-1)}, \frac{\Delta_{2}}{\Delta \Gamma(\beta-1)} \int_{0}^{1} a(\tau) d A(\tau)+\frac{1}{\Gamma(\beta-1)},\right.
$$




$$
\begin{aligned}
& \left.\frac{1}{\Delta \Gamma(\alpha-1)} \int_{0}^{1} \mathrm{~b}(\tau) \mathrm{dB}(\tau), \frac{1}{\Delta \Gamma(\beta-1)} \int_{0}^{1} \mathrm{a}(\tau) \mathrm{dA}(\tau)\right\}, \\
\mathrm{k}_{2}=\min & \left\{\frac{\Delta_{1}}{\Delta \Gamma(\alpha)} \int_{0}^{1} \tau^{\alpha-1}(1-\tau) \mathrm{b}(\tau) \mathrm{dB}(\tau), \frac{\Delta_{2}}{\Delta \Gamma(\beta)} \int_{0}^{1} \tau^{\beta-1}(1-\tau) \mathrm{a}(\tau) \mathrm{d} A(\tau),\right. \\
& \left.\frac{1}{\Delta \Gamma(\alpha)} \int_{0}^{1} \tau^{\alpha-1}(1-\tau) \mathrm{b}(\tau) \mathrm{dB}(\tau), \frac{1}{\Delta \Gamma(\beta)} \int_{0}^{1} \tau^{\beta-1}(1-\tau) \mathrm{a}(\tau) \mathrm{dA}(\tau)\right\},
\end{aligned}
$$

and $\gamma_{1}=\min \{\alpha-1, \beta-1\}, \gamma_{2}=\max \{\alpha-1, \beta-1\}$.

Proof. For any $t, s \in[0,1]$, by $(2.2),(2.4),(2.5)$ and Lemma 2.6, we get

$$
\begin{aligned}
\mathrm{G}_{1}(\mathrm{t}, \mathrm{s}) & =\mathrm{g}_{1}(\mathrm{t}, \mathrm{s})+\frac{\Delta_{1}}{\Delta} \mathrm{t}^{\alpha-1} \int_{0}^{1} \mathrm{~g}_{1}(\tau, \mathrm{s}) \mathrm{b}(\tau) \mathrm{dB}(\tau) \\
& \leqslant \frac{s(1-s)^{\alpha-1}}{\Gamma(\alpha-1)}+\frac{\Delta_{1}}{\Delta} \mathrm{t}^{\alpha-1} \int_{0}^{1} \mathrm{~g}_{1}(\tau, s) \mathrm{b}(\tau) \mathrm{dB}(\tau) \\
& \leqslant \frac{s(1-s)^{\alpha-1}}{\Gamma(\alpha-1)}+\frac{\Delta_{1} s(1-s)^{\alpha-1}}{\Delta \Gamma(\alpha-1)} \int_{0}^{1} \mathrm{~b}(\tau) \mathrm{dB}(\tau) \\
& =\left(\frac{\Delta_{1}}{\Delta \Gamma(\alpha-1)} \int_{0}^{1} \mathrm{~b}(\tau) \mathrm{dB}(\tau)+\frac{1}{\Gamma(\alpha-1)}\right) s(1-s)^{\alpha-1},
\end{aligned}
$$

or

$$
\begin{aligned}
G_{1}(t, s) & \leqslant \frac{t^{\alpha-1}(1-t)^{\alpha-1}}{\Gamma(\alpha)}+\frac{\Delta_{1}}{\Delta} t^{\alpha-1} \int_{0}^{1} \frac{t^{\alpha-1}(1-t)^{\alpha-1}}{\Gamma(\alpha)} b(\tau) d B(\tau) \\
& \leqslant \frac{t^{\alpha-1}}{\Gamma(\alpha)}+\frac{\Delta_{1}}{\Delta \Gamma(\alpha-1)} t^{\alpha-1} \int_{0}^{1} b(\tau) d B(\tau) \\
& \leqslant k_{1} t^{\alpha-1} .
\end{aligned}
$$

In a similar way, we can get

$$
\begin{aligned}
\mathrm{G}_{2}(\mathrm{t}, \mathrm{s}) & =\mathrm{g}_{2}(\mathrm{t}, \mathrm{s})+\frac{\Delta_{2}}{\Delta} \mathrm{t}^{\beta-1} \int_{0}^{1} g_{2}(\tau, s) \mathrm{a}(\tau) \mathrm{dA}(\tau) \\
& \leqslant\left(\frac{\Delta_{2}}{\Delta \Gamma(\beta-1)} \int_{0}^{1} \mathrm{a}(\tau) \mathrm{dA}(\tau)+\frac{1}{\Gamma(\beta-1)}\right) \mathrm{s}(1-\mathrm{s})^{\beta-1},
\end{aligned}
$$

or

$$
G_{2}(t, s) \leqslant k_{1} t^{\beta-1}
$$

On the other hand, we have

$$
\begin{aligned}
\mathrm{G}_{1}(\mathrm{t}, \mathrm{s}) & =g_{1}(\mathrm{t}, \mathrm{s})+\frac{\Delta_{1}}{\Delta} \mathrm{t}^{\alpha-1} \int_{0}^{1} g_{1}(\tau, \mathrm{s}) \mathrm{b}(\tau) \mathrm{dB}(\tau) \\
& \geqslant \frac{\Delta_{1}}{\Delta} \mathrm{t}^{\alpha-1} \int_{0}^{1} \frac{\tau^{\alpha-1}(1-\tau) s(1-s)^{\alpha-1}}{\Gamma(\alpha)} \mathrm{b}(\tau) \mathrm{dB}(\tau) \\
& =\frac{\Delta_{1}}{\Delta \Gamma(\alpha)} \int_{0}^{1} \tau^{\alpha-1}(1-\tau) \mathrm{b}(\tau) \mathrm{dB}(\tau) \mathrm{t}^{\alpha-1} s(1-\mathrm{s})^{\alpha-1}
\end{aligned}
$$

In a similar way, we get

$$
\mathrm{G}_{2}(\mathrm{t}, \mathrm{s}) \geqslant \frac{\Delta_{2}}{\Delta \Gamma(\beta)} \int_{0}^{1} \tau^{\beta-1}(1-\tau) \mathrm{a}(\tau) \mathrm{dA}(\tau) \mathrm{t}^{\beta-1} \mathrm{~s}(1-\mathrm{s})^{\beta-1} .
$$


In the same way, we obtain the other inequalities about $H_{i}(t, s)(i=1,2)$, so we omit it. The proof is complete.

For convenience in presentation, we present the assumptions to be used later in the following.

$\left(\mathrm{H}_{0}\right) \quad A, B:[0,1] \rightarrow \mathbb{R}$ are functions of bounded variation and $\int_{0}^{1} g_{i}(t, s) b(t) d B(t)>0, \int_{0}^{1} g_{i}(t, s) a(t) d A(t)>$ $0(i=1,2)$ for all $s \in[0,1]$;

$\left(H_{1}\right) \quad f_{i} \in C((0,1) \times(0, \infty) \times(0, \infty),[0, \infty))$ may be singular at $t=0,1$ and $y=0, f_{i}(t, x, y)$ is nondecreasing in $x$ and nonincreasing in $y$, and there exist $\lambda_{i}, \mu_{i} \in[0,1)$ such that

$$
c^{\lambda_{i}} f_{i}(t, x, y) \leqslant f_{i}(t, c x, y), \quad f_{i}(t, x, c y) \leqslant c^{-\mu_{i}} f_{i}(t, x, y), \quad \forall x, y>0, c \in(0,1), i=1,2 .
$$

$\left(\mathrm{H}_{2}\right) \mathrm{g}_{\mathrm{i}} \in \mathrm{C}((0,1) \times(0, \infty) \times(0, \infty),[0, \infty))$ may be singular at $\mathrm{t}=0,1$ and $x=0, g_{i}(t, x, y)$ is nonincreasing in $x$ and nondecreasing in $y$, and there exist $\xi_{i}, \eta_{i} \in[0,1)$ such that

$$
c^{\xi_{i}} g_{i}(t, x, y) \leqslant g_{i}(t, x, c y), \quad g_{i}(t, c x, y) \leqslant c^{-\eta_{i}} g_{i}(t, x, y), \quad \forall x, y>0, c \in(0,1), i=1,2 .
$$

$\left(H_{3}\right) 0<\int_{0}^{1} p_{i}(t) f_{i}\left(t, 1, t^{\gamma_{2}}\right) d t<\infty, 0<\int_{0}^{1} q_{i}(t) g_{i}\left(t, t^{\gamma_{2}}, 1\right) d t<\infty, i=1,2$.

Remark 2.8.

(1) $\left(\mathrm{H}_{1}\right)$ implies that

$$
f_{i}(t, c x, y) \leqslant c^{\lambda_{i}} f_{i}(t, x, y), \quad f_{i}(t, x, c y) \leqslant c^{\mu_{i}} f_{i}(t, x, y), \quad \forall x, y>0, \quad c>1, \quad i=1,2
$$

(2) $\left(\mathrm{H}_{2}\right)$ implies that

$$
g_{i}(t, x, c y) \leqslant c^{\xi_{i}} g_{i}(t, x, y), \quad g_{i}(t, x, y) \leqslant c^{\eta_{i}} g_{i}(t, c x, y), \quad \forall x, y>0, \quad c>1, \quad i=1,2 .
$$

Remark 2.9. By $\left(\mathrm{H}_{1}\right),\left(\mathrm{H}_{2}\right)$ and $\left(\mathrm{H}_{3}\right)$, we can get

$$
0<\int_{0}^{1} p_{i}(t) f_{i}\left(t, t^{\gamma_{2}}, 1\right) d t<\infty, \quad 0<\int_{0}^{1} q_{i}(t) g_{i}\left(t, 1, t^{\gamma_{2}}\right) d t<\infty, \quad i=1,2 .
$$

For our constructions, we shall consider the Banach space $E=C[0,1]$ equipped with the standard norm $\|u\|=\max _{t \in[0,1]}|u(t)|$. Let $Q=\{u \in E \mid u(t) \geqslant 0, t \in[0,1]\}$, $Q$ is a cone of $E$. Similarly, for each $(x, y) \in E \times E$, we write $\|(x, y)\|_{1}=\max \{\|x\|,\|y\|\}$. It is easy to see that $\left(E \times E,\|\cdot\|_{1}\right)$ is a Banach space. We define a cone $P$ of $E \times E$ by

$$
P=\left\{(x, y) \in E \times E: x(t) \geqslant k t^{\gamma_{2}}\|(x, y)\|_{1}, y(t) \geqslant k t^{\gamma_{2}}\|(x, y)\|_{1}, t \in[0,1]\right\},
$$

where $k=\frac{k_{1}}{k_{2}} \in(0,1)$, in which $k_{1}$ and $k_{2}$ are defined by Lemma 2.7. For any $r>0$, let $P_{r}=\{(x, y) \in P$ : $\left.\|(x, y)\|_{1}<r\right\}, \partial P_{r}=\left\{(x, y) \in P:\|(x, y)\|_{1}=r\right\}$.

Define an operator $\mathrm{T}: \mathrm{P} \backslash\{\theta\} \rightarrow \mathrm{E} \times \mathrm{E}$ by

$$
T(x, y)=\left(T_{1}(x, y), T_{2}(x, y)\right),
$$

where the operators $T_{1}, T_{2}: P \backslash\{\theta\} \rightarrow Q$ are defined by

$$
\begin{aligned}
T_{1}(x, y)(t)= & \int_{0}^{1} G_{1}(t, s)\left[p_{1}(s) f_{1}(s, x(s), y(s))+q_{1}(s) g_{1}(s, x(s), y(s))\right] d s \\
& +\int_{0}^{1} H_{1}(t, s)\left[p_{2}(s) f_{2}(s, x(s), y(s))+q_{2}(s) g_{2}(s, x(s), y(s))\right] d s \\
T_{2}(x, y)(t)= & \int_{0}^{1} G_{2}(t, s)\left[p_{2}(s) f_{2}(s, x(s), y(s))+q_{2}(s) g_{2}(s, x(s), y(s))\right] d s \\
& +\int_{0}^{1} H_{2}(t, s)\left[p_{1}(s) f_{1}(s, x(s), y(s))+q_{1}(s) g_{1}(s, x(s), y(s))\right] d s
\end{aligned}
$$


Lemma 2.10. Assume that $\left(\mathrm{H}_{1}\right)$ and $\left(\mathrm{H}_{2}\right)$ hold. Then, for any $0<\mathrm{r}<\mathrm{R}<+\infty, \mathrm{T}:\left(\overline{\mathrm{P}_{\mathrm{R}}} \backslash \mathrm{P}_{\mathrm{r}}\right) \rightarrow \mathrm{P}$ is a completely continuous operator.

Proof. Firstly, we claim that $T(x, y)$ is well-defined for $(x, y) \in P \backslash\{\theta\}$. In fact, since $(x, y) \in P \backslash\{\theta\}$, we can see that

$$
x(t) \geqslant k t^{\gamma_{2}}\|(x, y)\|_{1}>0, \quad y(t) \geqslant k t^{\gamma_{2}}\|(x, y)\|_{1}>0, \quad t \in(0,1] .
$$

Let $c$ be a positive number such that $c>1$ and $\|(x, y)\|_{1} / c<1$. From $\left(H_{1}\right),\left(H_{2}\right)$ and Remark 2.8, we have

$$
\begin{aligned}
f_{i}(t, x(t), y(t)) & \leqslant f_{i}\left(t, c, k t^{\gamma_{2}}\|(x, y)\|_{1}\right) \\
& \leqslant c^{\lambda_{i}} f_{i}\left(t, 1, \frac{k t^{\gamma_{2}}\|(x, y)\|_{1}}{c}\right) \\
& \leqslant c^{\lambda_{i}}\left(\frac{k\|(x, y)\|_{1}}{c}\right)^{-\mu_{i}} f\left(t, 1, t^{\gamma_{2}}\right) \\
& =c^{\lambda_{i}+\mu_{i}}\left(k\|(x, y)\|_{1}\right)^{-\mu_{i}} f_{i}\left(t, 1, t^{\gamma_{2}}\right), \quad i=1,2, \\
g_{i}(t, x(t), y(t)) & \leqslant c^{\xi_{i}+\eta_{i}}\left(k\|(x, y)\|_{1}\right)^{-\eta_{i}} g_{i}\left(t, t^{\gamma_{2}}, 1\right), \quad i=1,2 .
\end{aligned}
$$

Hence, for any $t \in[0,1]$, we get

$$
\begin{aligned}
\mathrm{T}_{1}(x, y)(t) \leqslant & k_{1} \int_{0}^{1} p_{1}(s) f_{1}(s, x(s), y(s))+q_{1}(s) g_{1}(s, x(s), y(s)) d s \\
& +k_{1} \int_{0}^{1} p_{2}(s) f_{2}(s, x(s), y(s))+q_{2}(s) g_{2}(s, x(s), y(s)) d s \\
\leqslant & k_{1} c^{\lambda_{1}+\mu_{1}}\left(k\|(x, y)\|_{1}\right)^{-\mu_{1}} \int_{0}^{1} p_{1}(s) f_{1}\left(s, 1, s^{\gamma_{2}}\right) d s \\
& +k_{1} c^{\xi_{1}+\eta_{1}}\left(k\|(x, y)\|_{1}\right)^{-\eta_{1}} \int_{0}^{1} q_{1}(s) g_{1}\left(s, s^{\gamma_{2}}, 1\right) d s \\
& +k_{1} c^{\lambda_{2}+\mu_{2}}\left(k\|(x, y)\|_{1}\right)^{-\mu_{2}} \int_{0}^{1} p_{2}(s) f_{2}\left(s, 1, s^{\gamma_{2}}\right) d s \\
& +k_{1} c^{\xi_{2}+\eta_{2}}\left(k\|(x, y)\|_{1}\right)^{-\eta_{2}} \int_{0}^{1} q_{2}(s) g_{2}\left(s, s^{\gamma_{2}}, 1\right) d s \\
< &
\end{aligned}
$$

Similarly, we can prove $T_{2}(x, y)(t)<\infty$. Thus we can say that $T$ is well-defined on $P \backslash\{\theta\}$.

Secondly, we show that $T\left(\overline{P_{R}} \backslash P_{r}\right) \subset P$. By Lemma 2.7 , for all $\tau, t, s \in[0,1]$, we obtain

$$
\begin{array}{ll}
\mathrm{G}_{1}(t, s) \geqslant k t^{\alpha-1} \mathrm{G}_{1}(\tau, s), & \mathrm{G}_{2}(t, s) \geqslant k t^{\beta-1} \mathrm{G}_{2}(\tau, s), \\
\mathrm{H}_{1}(t, s) \geqslant k t^{\alpha-1} \mathrm{H}_{1}(\tau, s), & \mathrm{H}_{2}(t, s) \geqslant k t^{\beta-1} \mathrm{H}_{2}(\tau, s), \\
\mathrm{H}_{1}(t, s) \geqslant k t^{\alpha-1} \mathrm{G}_{2}(\tau, s), & \mathrm{G}_{1}(t, s) \geqslant k t^{\alpha-1} \mathrm{H}_{2}(\tau, s), \\
\mathrm{H}_{2}(t, s) \geqslant k t^{\beta-1} \mathrm{G}_{1}(\tau, s), & \mathrm{G}_{2}(t, s) \geqslant k t^{\beta-1} \mathrm{H}_{1}(\tau, s) .
\end{array}
$$

Hence, for $(x, y) \in\left(\overline{P_{R}} \backslash P_{r}\right), t \in[0,1]$, we have

$$
\begin{aligned}
T_{1}(x, y)(t) \geqslant & k t^{\alpha-1} \int_{0}^{1} G_{1}(\tau, s)\left[p_{1}(s) f_{1}(s, x(s), y(s))+q_{1}(s) g_{1}(s, x(s), y(s))\right] d s \\
& +k t^{\alpha-1} \int_{0}^{1} H_{1}(\tau, s)\left[p_{2}(s) f_{2}(s, x(s), y(s))+q_{2}(s) g_{2}(s, x(s), y(s))\right] d s \\
\geqslant & k t^{\gamma^{2}} T_{1}(x, y)(\tau), \quad \forall \tau \in[0,1],
\end{aligned}
$$




$$
\begin{aligned}
\mathrm{T}_{1}(x, y)(t) \geqslant & k t^{\alpha-1} \int_{0}^{1} \mathrm{H}_{2}(\tau, s)\left[p_{1}(s) f_{1}(s, x(s), y(s))+q_{1}(s) g_{1}(s, x(s), y(s))\right] d s \\
& +k t^{\alpha-1} \int_{0}^{1} G_{2}(\tau, s)\left[p_{2}(s) f_{2}(s, x(s), y(s))+q_{2}(s) g_{2}(s, x(s), y(s))\right] d s \\
\geqslant & k t^{\gamma_{2}} T_{2}(x, y)(\tau), \quad \forall \tau \in[0,1] .
\end{aligned}
$$

Then, $T_{1}(x, y)(t) \geqslant k t^{\gamma_{2}}\left\|T_{1}(x, y)\right\|$ and $T_{1}(x, y)(t) \geqslant k t^{\gamma_{2}}\left\|T_{2}(x, y)\right\|$, that is,

$$
\mathrm{T}_{1}(x, y)(t) \geqslant k t^{\gamma_{2}}\left\|\left(\mathrm{~T}_{1}(x, y), \mathrm{T}_{2}(x, y)\right)\right\|_{1} .
$$

In the same way, we can prove that

$$
T_{2}(x, y)(t) \geqslant k t^{\gamma_{2}}\left\|\left(T_{1}(x, y), T_{2}(x, y)\right)\right\|_{1} .
$$

Therefore, $T\left(\overline{P_{R}} \backslash P_{r}\right) \subset P$.

Next, we prove that $T$ is a compact operator. Suppose $V \subset \overline{P_{R}} \backslash P_{r}$ is an arbitrary bounded set in $E \times E$. Then from the above proof, we know that $T(V)$ is uniformly bounded. In the following, we shall show that $T(V)$ is equicontinuous on $[0,1]$. For all $(x, y) \in V, t \in[0,1]$, by using Lemma 2.5, we have

$$
\begin{aligned}
& T_{1}(x, y)(t)=\int_{0}^{1} G_{1}(t, s)\left[p_{1}(s) f_{1}(s, x(s), y(s))+q_{1}(s) g_{1}(s, x(s), y(s))\right] d s \\
& +\int_{0}^{1} H_{1}(t, s)\left[p_{2}(s) f_{2}(s, x(s), y(s))+q_{2}(s) g_{2}(s, x(s), y(s))\right] d s \\
& =\frac{\Delta_{1}}{\Delta} \mathrm{t}^{\alpha-1} \int_{0}^{1}\left(\int_{0}^{1} g_{1}(\tau, s) b(\tau) d B(\tau)\right)\left[p_{1}(s) f_{1}(s, x(s), y(s))\right. \\
& \left.+q_{1}(s) g_{1}(s, x(s), y(s))\right] d s \\
& +\int_{0}^{t} \frac{t^{\alpha-1}(1-s)^{\alpha-1}-(t-s)^{\alpha-1}}{\Gamma(\alpha)}\left[p_{1}(s) f_{1}(s, x(s), y(s))\right. \\
& \left.+q_{1}(s) g_{1}(s, x(s), y(s))\right] d s \\
& +\int_{t}^{1} \frac{t^{\alpha-1}(1-s)^{\alpha-1}}{\Gamma(\alpha)}\left[p_{1}(s) f_{1}(s, x(s), y(s))\right. \\
& \left.+\mathrm{q}_{1}(\mathrm{~s}) \mathrm{g}_{1}(\mathrm{~s}, x(\mathrm{~s}), \mathrm{y}(\mathrm{s}))\right] \mathrm{d} s \\
& +\frac{t^{\alpha-1}}{\Delta} \int_{0}^{1}\left(\int_{0}^{1} g_{2}(\tau, s) a(\tau) d A(\tau)\right)\left[p_{2}(s) f_{2}(s, x(s), y(s))\right. \\
& \left.+q_{2}(s) g_{2}(s, x(s), y(s))\right] d s \text {. }
\end{aligned}
$$

Differentiating the above formula with respect to $t$ and combining $\left(\mathrm{H}_{1}\right)$ and $\left(\mathrm{H}_{2}\right)$, we obtain

$$
\begin{aligned}
& \left|\mathrm{T}_{1}(x, y)^{\prime}(\mathrm{t})\right|=\frac{(\alpha-1) \Delta_{1}}{\Delta} \mathrm{t}^{\alpha-2} \int_{0}^{1}\left(\int_{0}^{1} g_{1}(\tau, s) b(\tau) d B(\tau)\right)\left[p_{1}(s) f_{1}(s, x(s), y(s))\right. \\
& \left.+q_{1}(s) g_{1}(s, x(s), y(s))\right] d s \\
& +\int_{0}^{t} \frac{(\alpha-1) t^{\alpha-2}(1-s)^{\alpha-1}-(\alpha-1)(t-s)^{\alpha-2}}{\Gamma(\alpha)}\left[p_{1}(s) f_{1}(s, x(s), y(s))\right. \\
& \left.+\mathrm{q}_{1}(\mathrm{~s}) \mathrm{g}_{1}(\mathrm{~s}, x(\mathrm{~s}), \mathrm{y}(\mathrm{s}))\right] \mathrm{ds} \\
& +\int_{t}^{1} \frac{(\alpha-1) t^{\alpha-2}(1-s)^{\alpha-1}}{\Gamma(\alpha)}\left[p_{1}(s) f_{1}(s, x(s), y(s))\right. \\
& \left.+q_{1}(s) g_{1}(s, x(s), y(s))\right] d s
\end{aligned}
$$




$$
\begin{aligned}
& +\frac{(\alpha-1) t^{\alpha-2}}{\Delta} \int_{0}^{1}\left(\int_{0}^{1} g_{2}(\tau, s) a(\tau) d A(\tau)\right)\left[p_{2}(s) f_{2}(s, x(s), y(s))\right. \\
& \left.+\mathrm{q}_{2}(\mathrm{~s}) \mathrm{g}_{2}(\mathrm{~s}, x(\mathrm{~s}), \mathrm{y}(\mathrm{s}))\right] \mathrm{ds} \\
& \leqslant \frac{(\alpha-1) \Delta_{1}}{\Delta} \int_{0}^{1}\left(\int_{0}^{1} g_{1}(\tau, s) b(\tau) d B(\tau)\right)\left[p_{1}(s) f_{1}(s, x(s), y(s))\right. \\
& \left.+q_{1}(s) g_{1}(s, x(s), y(s))\right] d s \\
& +\int_{0}^{t} \frac{(\alpha-1) t^{\alpha-2}(1-s)^{\alpha-1}-(\alpha-1)(t-s)^{\alpha-2}}{\Gamma(\alpha)}\left[p_{1}(s) f_{1}(s, x(s), y(s))\right. \\
& \left.+\mathrm{q}_{1}(\mathrm{~s}) \mathrm{g}_{1}(\mathrm{~s}, x(\mathrm{~s}), \mathrm{y}(\mathrm{s}))\right] \mathrm{ds} \\
& +\int_{t}^{1} \frac{(\alpha-1) t^{\alpha-2}(1-s)^{\alpha-1}}{\Gamma(\alpha)}\left[p_{1}(s) f_{1}(s, x(s), y(s))\right. \\
& \left.+\mathrm{q}_{1}(\mathrm{~s}) \mathrm{g}_{1}(\mathrm{~s}, x(\mathrm{~s}), \mathrm{y}(\mathrm{s}))\right] \mathrm{ds} \\
& +\frac{(\alpha-1) t^{\alpha-2}}{\Delta} \int_{0}^{1}\left(\int_{0}^{1} g_{2}(\tau, s) a(\tau) d A(\tau)\right)\left[p_{2}(s) f_{2}(s, x(s), y(s))\right. \\
& \left.+q_{2}(s) g_{2}(s, x(s), y(s))\right] d s \\
& \leqslant(\alpha-1) k_{1} c^{\lambda_{1}+\mu_{1}}\left(k\|(x, y)\|_{1}\right)^{-\mu_{1}} \int_{0}^{1} p_{1}(s) f_{1}\left(s, 1, s^{\gamma_{2}}\right) d s \\
& +(\alpha-1) k_{1} c^{\xi_{1}+\eta_{1}}\left(k\|(x, y)\|_{1}\right)^{-\eta_{1}} \int_{0}^{1} q_{1}(s) g_{1}\left(s, s^{\gamma_{2}}, 1\right) d s \\
& +k_{1} c^{\lambda_{1}+\mu_{1}}\left(k\|(x, y)\|_{1}\right)^{-\mu_{1}} \int_{0}^{t} \frac{(\alpha-1) t^{\alpha-2}(1-s)^{\alpha-1}-(\alpha-1)(t-s)^{\alpha-2}}{\Gamma(\alpha)} \\
& p_{1}(s) f_{1}\left(s, 1, s^{\gamma_{2}}\right) d s \\
& +k_{1} c^{\xi_{1}+\eta_{1}}\left(k\|(x, y)\|_{1}\right)^{-\eta_{1}} \int_{0}^{t} \frac{(\alpha-1) t^{\alpha-2}(1-s)^{\alpha-1}-(\alpha-1)(t-s)^{\alpha-2}}{\Gamma(\alpha)} \\
& \mathrm{q}_{1}(\mathrm{~s}) \mathrm{g}_{1}\left(\mathrm{~s}, \mathrm{~s}^{\gamma_{2}}, 1\right) \mathrm{d} s \\
& +k_{1} c^{\lambda_{1}+\mu_{1}}\left(k\|(x, y)\|_{1}\right)^{-\mu_{1}} \int_{t}^{1} \frac{(\alpha-1) t^{\alpha-2}(1-s)^{\alpha-1}}{\Gamma(\alpha)} p_{1}(s) f_{1}\left(s, 1, s^{\gamma_{2}}\right) d s \\
& +k_{1} c^{\xi_{1}+\eta_{1}}\left(k\|(x, y)\|_{1}\right)^{-\eta_{1}} \int_{t}^{1} \frac{(\alpha-1) t^{\alpha-2}(1-s)^{\alpha-1}}{\Gamma(\alpha)} q_{1}(s) g_{1}\left(s, s^{\gamma_{2}}, 1\right) d s \\
& +(\alpha-1) k_{1} c^{\lambda_{2}+\mu_{2}}\left(k\|(x, y)\|_{1}\right)^{-\mu_{2}} \int_{0}^{1} p_{2}(s) f_{2}\left(s, 1, s^{\gamma_{2}}\right) d s \\
& +(\alpha-1) k_{1} c^{\xi_{2}+\eta_{2}}\left(k\|(x, y)\|_{1}\right)^{-\eta_{2}} \int_{0}^{1} q_{2}(s) g_{2}\left(s, s^{\gamma_{2}}, 1\right) d s \\
& \leqslant c^{\lambda_{1}+\mu_{1}}(k r)^{-\mu_{1}}\left[(\alpha-1) k_{1} \int_{0}^{1} p_{1}(s) f_{1}\left(s, 1, s^{\gamma_{2}}\right) d s\right. \\
& +\int_{0}^{t} \frac{(\alpha-1) t^{\alpha-2}(1-s)^{\alpha-1}-(\alpha-1)(t-s)^{\alpha-2}}{\Gamma(\alpha)} p_{1}(s) f_{1}\left(s, 1, s^{\gamma_{2}}\right) d s \\
& \left.+\int_{t}^{1} \frac{(\alpha-1) t^{\alpha-2}(1-s)^{\alpha-1}}{\Gamma(\alpha)} p_{1}(s) f_{1}\left(s, 1, s^{\gamma_{2}}\right) d s\right] \\
& +c^{\xi_{1}+\eta_{1}}(k r)^{-\eta_{1}}\left[(\alpha-1) k_{1} \int_{0}^{1} q_{1}(s) g_{1}\left(s, s^{\gamma_{2}}, 1\right) d s\right.
\end{aligned}
$$




$$
\begin{aligned}
& +\int_{0}^{t} \frac{(\alpha-1) t^{\alpha-2}(1-s)^{\alpha-1}-(\alpha-1)(t-s)^{\alpha-2}}{\Gamma(\alpha)} q_{1}(s) g_{1}\left(s, s^{\gamma_{2}}, 1\right) d s \\
& \left.+\int_{t}^{1} \frac{(\alpha-1) t^{\alpha-2}(1-s)^{\alpha-1}}{\Gamma(\alpha)} q_{1}(s) g_{1}\left(s, s^{\gamma_{2}}, 1\right) d s\right] \\
& +(\alpha-1) k_{1} c^{\lambda_{2}+\mu_{2}}(k r)^{-\mu_{2}} \int_{0}^{1} p_{2}(s) f_{2}\left(s, 1, s^{\gamma_{2}}\right) d s \\
& +(\alpha-1) k_{1} c^{\xi_{2}+\eta_{2}}(k r)^{-\eta_{2}} \int_{0}^{1} q_{2}(s) g_{2}\left(s, s^{\gamma_{2}}, 1\right) d s=: K(t) .
\end{aligned}
$$

Exchanging the integration order, we have

$$
\begin{aligned}
& \int_{0}^{1} K(t) d t=c^{\lambda_{1}+\mu_{1}}(k r)^{-\mu_{1}}\left[(\alpha-1) k_{1} \int_{0}^{1} p_{1}(s) f_{1}\left(s, 1, s^{\gamma_{2}}\right) d s\right] \\
& +c^{\xi_{1}+\eta_{1}}(k r)^{-\eta_{1}}\left[(\alpha-1) k_{1} \int_{0}^{1} q_{1}(s) g_{1}\left(s, s^{\gamma_{2}}, 1\right) d s\right] \\
& +(\alpha-1) k_{1} c^{\lambda_{2}+\mu_{2}}(k r)^{-\mu_{2}} \int_{0}^{1} p_{2}(s) f_{2}\left(s, 1, s^{\gamma_{2}}\right) d s \\
& +(\alpha-1) k_{1} c^{\xi_{2}+\eta_{2}}(k r)^{-\eta_{2}} \int_{0}^{1} q_{2}(s) g_{2}\left(s, s^{\gamma_{2}}, 1\right) d s \\
& <+\infty \text {. }
\end{aligned}
$$

From the absolute continuity of the integral, we know that $T_{1}(V)$ is equicontinuous on $[0,1]$. Thus, according to the Ascoli-Arzela theorem, $\mathrm{T}_{1}(\mathrm{~V})$ is a relatively compact set. In the same way, we can prove that $\mathrm{T}_{2}(\mathrm{~V})$ is a relatively compact set. Therefore, $\mathrm{T}(\mathrm{V})$ is relatively compact.

Finally, we prove that $T:\left(\overline{P_{R}} \backslash P_{r}\right) \rightarrow Q$ is continuous. We need to prove only $T_{1}, T_{2}:\left(\overline{P_{R}} \backslash P_{r}\right) \rightarrow Q$ are continuous. Suppose that $\left(x_{n}, y_{n}\right),\left(x_{0}, y_{0}\right) \in \overline{P_{R}} \backslash P_{r}$ and $\left\|\left(x_{n}, y_{n}\right)-\left(x_{0}, y_{0}\right)\right\|_{1} \rightarrow 0(n \rightarrow \infty)$. Let $S=\sup \left\{\left\|\left(x_{n}, y_{n}\right)\right\|_{1} \mid n=0,1,2, \cdots\right\}$. We choose a positive constant $M$ such that $S / M<1$ and $M>1$. From (2.12) and (2.13), for any $t \in(0,1)$, we know

$$
\begin{aligned}
& f_{i}\left(t, x_{n}(t), y_{n}(t)\right) \leqslant M^{\lambda_{i}+\mu_{i}}(k r)^{-\mu_{i}} f_{i}\left(t, 1, t^{\gamma_{2}}\right), \quad n=0,1,2, \cdots, \quad i=1,2, \\
& g_{i}\left(t, x_{n}(t), y_{n}(t)\right) \leqslant M^{\xi_{i}+\eta_{i}}(k r)^{-\eta_{i}} g_{i}\left(t, t^{\gamma_{2}}, 1\right), \quad n=0,1,2, \cdots, \quad i=1,2 .
\end{aligned}
$$

Then by Lemma 2.7 , for any $t \in[0,1]$, we get

$$
\begin{aligned}
\left|T_{1}\left(x_{n}, y_{n}\right)(t)-T_{1}\left(x_{0}, y_{0}\right)(t)\right| \leqslant & k_{1} \int_{0}^{1}\left[\left|p_{1}(s) \| f_{1}\left(s, x_{n}(s), y_{n}(s)\right)-f_{1}\left(s, x_{0}(s), y_{0}(s)\right)\right|\right. \\
& \left.+\left|q_{1}(s) \| g_{1}\left(s, x_{n}(s), y_{n}(s)\right)-g_{1}\left(s, x_{0}(s), y_{0}(s)\right)\right|\right] d s \\
& +k_{1} \int_{0}^{1}\left[\left|p_{2}(s) \| f_{2}\left(s, x_{n}(s), y_{n}(s)\right)-f_{2}\left(s, x_{0}(s), y_{0}(s)\right)\right|\right. \\
& \left.+\left|q_{2}(s)\right|\left|g_{2}\left(s, x_{n}(s), y_{n}(s)\right)-g_{2}\left(s, x_{0}(s), y_{0}(s)\right)\right|\right] d s
\end{aligned}
$$

For any $\epsilon>0$, by $\left(\mathrm{H}_{3}\right)$, there exists a positive number $\delta \in\left(0, \frac{1}{2}\right)$ such that

$$
\begin{aligned}
& \int_{H_{(\delta)}} k_{1} M^{\lambda_{i}+\mu_{i}}(k r)^{-\mu_{i}} p_{i}(s) f_{i}\left(t, 1, t^{\gamma_{2}}\right) d s<\frac{\epsilon}{4}, \\
& \int_{H_{(\delta)}} k_{1} M^{\xi_{i}+\eta_{i}}(k r)^{-\eta_{i}} q_{i}(s) g_{i}\left(t, t^{\gamma_{2}}, 1\right) d s<\frac{\epsilon}{4},
\end{aligned}
$$


where $H_{(\delta)}=[0, \delta] \cup[1-\delta, 1]$. On the other hand, for $(x, y) \in \overline{P_{R}} \backslash P_{r}$ and $t \in[\delta, 1-\delta]$, we have

$$
0<r k \delta \leqslant x(t), \quad y(t) \leqslant R .
$$

Since $f_{i}(t, x, y)$ and $g_{i}(t, x, y)(i=1,2)$ are uniformly continuous in $[\delta, 1-\delta] \times[r k \delta, b] \times[r k \delta, b]$, we have

$$
\begin{aligned}
\lim _{n \rightarrow+\infty}\left|f_{i}\left(s, x_{n}(s), y_{n}(s)\right)-f_{i}\left(s, x_{0}(s), y_{0}(s)\right)\right| \\
\quad=\lim _{n \rightarrow+\infty}\left|g_{i}\left(s, x_{n}(s), y_{n}(s)\right)-g_{i}\left(s, x_{0}(s), y_{0}(s)\right)\right| \\
\quad=0,
\end{aligned}
$$

holds uniformly on $[\delta, 1-\delta]$ for $s$. Then the Lebesgue dominated convergence theorem yields that

$$
\begin{aligned}
& \int_{\delta}^{1-\delta}\left|p_{\mathfrak{i}}(s)\right|\left|f_{\mathfrak{i}}\left(s, x_{n}(s), y_{n}(s)\right)-f_{\mathfrak{i}}\left(s, x_{0}(s), y_{0}(s)\right)\right| d s \rightarrow 0, \\
& \int_{\delta}^{1-\delta}\left|q_{i}(s)\right|\left|g_{\mathfrak{i}}\left(s, x_{n}(s), y_{n}(s)\right)-g_{\mathfrak{i}}\left(s, x_{0}(s), y_{0}(s)\right)\right| d s \rightarrow 0, \quad n \rightarrow \infty .
\end{aligned}
$$

Thus, for above $\epsilon>0$, there exists a natural number $N$ such that for $n>N$ we have

$$
\begin{aligned}
& k_{1} \int_{\delta}^{1-\delta}\left[\left|p_{1}(s)\right|\left|f_{1}\left(s, x_{n}(s), y_{n}(s)\right)-f_{1}\left(s, x_{0}(s), y_{0}(s)\right)\right|\right. \\
& \left.\quad+\left|q_{1}(s) \| g_{1}\left(s, x_{n}(s), y_{n}(s)\right)-g_{1}\left(s, x_{0}(s), y_{0}(s)\right)\right|\right] d s \\
& \quad+k_{1} \int_{\delta}^{1-\delta}\left[\left|p_{2}(s) \| f_{2}\left(s, x_{n}(s), y_{n}(s)\right)-f_{2}\left(s, x_{0}(s), y_{0}(s)\right)\right|\right. \\
& \left.\quad+\left|q_{2}(s) \| g_{2}\left(s, x_{n}(s), y_{n}(s)\right)-g_{2}\left(s, x_{0}(s), y_{0}(s)\right)\right|\right] d s<\frac{\epsilon}{2}
\end{aligned}
$$

It follows from (2.14), (2.15), (2.16), (2.17), (2.18), (2.19), (2.20) that when $n>N$

$$
\begin{aligned}
\| T_{1}\left(x_{n}, y_{n}\right)- & T_{1}\left(x_{0}, y_{0}\right) \| \\
\leqslant & k_{1} \int_{0}^{1}\left[\left|p_{1}(s) \| f_{1}\left(s, x_{n}(s), y_{n}(s)\right)-f_{1}\left(s, x_{0}(s), y_{0}(s)\right)\right|\right. \\
& \left.+\left|q_{1}(s) \| g_{1}\left(s, x_{n}(s), y_{n}(s)\right)-g_{1}\left(s, x_{0}(s), y_{0}(s)\right)\right|\right] d s \\
& +k_{1} \int_{0}^{1}\left[\left|p_{2}(s) \| f_{2}\left(s, x_{n}(s), y_{n}(s)\right)-f_{2}\left(s, x_{0}(s), y_{0}(s)\right)\right|\right. \\
& \left.+\left|q_{2}(s) \| g_{2}\left(s, x_{n}(s), y_{n}(s)\right)-g_{2}\left(s, x_{0}(s), y_{0}(s)\right)\right|\right] d s \\
\leqslant & k_{1} \int_{H(\delta)} M^{\lambda_{1}+\mu_{1}}(k r)^{-\mu_{1}} p_{1}(s) f_{1}\left(s, 1, s^{\gamma_{2}}\right)+c^{\xi_{1}+\eta_{1}}(k r)^{-\eta_{1}} q_{1}(s) g_{1}\left(s, s^{\gamma_{2}}, 1\right) d s \\
& +k_{1} \int_{H(\delta)} M^{\lambda_{2}+\mu_{2}}(k r)^{-\mu_{2}} p_{2}(s) f_{2}\left(s, 1, s^{\gamma_{2}}\right)+M^{\xi_{2}+\eta_{2}}(k r)^{-\eta_{2}} q_{2}(s) g_{2}\left(s, s^{\gamma_{2}}, 1\right) d s \\
& +k_{1} \int_{\delta}^{1-\delta}\left[\left|p_{1}(s) \| f_{1}\left(s, x_{n}(s), y_{n}(s)\right)-f_{1}\left(s, x_{0}(s), y_{0}(s)\right)\right|\right. \\
& \left.+\left|q_{1}(s) \| g_{1}\left(s, x_{n}(s), y_{n}(s)\right)-g_{1}\left(s, x_{0}(s), y_{0}(s)\right)\right|\right] d s \\
& +k_{1} \int_{\delta}^{1-\delta}\left[\left|p_{2}(s) \| f_{2}\left(s, x_{n}(s), y_{n}(s)\right)-f_{2}\left(s, x_{0}(s), y_{0}(s)\right)\right|\right. \\
& \left.+\left|q_{2}(s) \| g_{2}\left(s, x_{n}(s), y_{n}(s)\right)-g_{2}\left(s, x_{0}(s), y_{0}(s)\right)\right|\right] d s<\epsilon .
\end{aligned}
$$


This implies that $T_{1}:\left(\overline{P_{R}} \backslash P_{r}\right) \rightarrow Q$ is continuous. Similarly, we can prove that $T_{2}:\left(\overline{P_{R}} \backslash P_{r}\right) \rightarrow Q$ is continuous. So, $T:\left(\overline{P_{R}} \backslash P_{r}\right) \rightarrow Q$ is continuous. By summing up, we get that $T:\left(\overline{P_{R}} \backslash P_{r}\right) \rightarrow P$ is completely continuous.

To prove the main results, we need the following well-known fixed point theorem.

Lemma 2.11 ([5]). Let $\mathrm{P}$ be a positive cone in a Banach space $E, \Omega_{1}$ and $\Omega_{2}$ be two bounded open sets in $E$ such that $\theta \in \Omega_{1}$ and $\overline{\Omega_{1}} \subset \Omega_{2}, A: \mathrm{P} \cap\left(\overline{\Omega_{2}} \backslash \Omega_{1}\right) \rightarrow \mathrm{P}$ be a completely continuous operator, where $\theta$ denotes the zero element of $\mathrm{E}$ and $\mathrm{P}$ is a cone of $\mathrm{E}$. Suppose that one of the following two conditions holds:

(i) $\|A u\| \leqslant\|u\|, \forall u \in P \cap \partial \Omega_{1} ; \quad\|A u\| \geqslant\|u\|, \forall u \in P \cap \partial \Omega_{2} ;$

(ii) $\|A u\| \geqslant\|u\|, \forall u \in P \cap \partial \Omega_{1} ; \quad\|A u\| \leqslant\|u\|, \forall u \in P \cap \partial \Omega_{2}$.

Then $\mathrm{A}$ has a fixed point in $\mathrm{P} \cap\left(\overline{\Omega_{2}} \backslash \Omega_{1}\right)$.

\section{Main results}

In this section, we shall give sufficient conditions for the existence and uniqueness of a positive solution for the BVP (1.1).

Theorem 3.1. Assume that conditions $\left(\mathrm{H}_{0}\right)-\left(\mathrm{H}_{3}\right)$ hold. Then the BVP (1.1) has at least one positive solution $\left(x^{*}, y^{*}\right)$ and there exists a real number $0<\mathrm{m}<1$ such that

$$
m t^{\gamma_{1}} \leqslant x^{*}(t) \leqslant \frac{1}{m} t^{\gamma_{1}}, \quad m t^{\gamma_{1}} \leqslant y^{*}(t) \leqslant \frac{1}{m} t^{\gamma_{1}}, \quad t \in[0,1],
$$

where $\gamma_{1}=\min \{\alpha-1, \beta-1\}$

Proof. We first prove that the differential system (1.1) has at least one positive solution $\left(x^{*}, y^{*}\right)$. Choose $d$ and D such that

$$
\begin{aligned}
0<\mathrm{d} \leqslant \min _{i=1,2} & \left\{\left(( \frac { 1 } { 4 } ) ^ { \gamma _ { 2 } } \mathrm { k } _ { 2 } \mathrm { k } ^ { \operatorname { m a x } \{ \lambda _ { i } , \mu _ { i } \} } \int _ { 0 } ^ { 1 } s ( 1 - s ) ^ { \gamma _ { 2 } } \left(p_{i}(s) f_{i}\left(s, s^{\gamma_{2}}, 1\right)\right.\right.\right. \\
& \left.\left.\left.+q_{i}(s) g_{i}\left(s, 1, s^{\gamma_{2}}\right)\right) d s\right)^{\frac{1}{1-\max \left\{\lambda_{i}, \mu_{i}\right\}}}, \frac{1}{2}\right\}, \\
D \geqslant \max & \left\{\left[k _ { 1 } \left(\int_{0}^{1}\left(p_{1}(s) f_{1}\left(s, 1, s^{\gamma_{2}}\right)+q_{1}(s) g_{1}\left(s, s^{\gamma_{2}}, 1\right)\right) d s+\int_{0}^{1}\left(p_{2}(s) f_{2}\left(s, 1, s^{\gamma_{2}}\right)\right.\right.\right.\right. \\
& \left.\left.\left.\left.+q_{2}(s) g_{2}\left(s, s^{\gamma_{2}}, 1\right)\right) d s\right)\right]^{\frac{1}{1-\max \left\{\lambda_{1}, \lambda_{2}, \xi_{1}, \xi_{2}\right\}}}, \frac{1}{k}, 2\right\} .
\end{aligned}
$$

Clearly $0<\mathrm{d}<1<\mathrm{D}$. By Lemma $2.10, \mathrm{~T}: \overline{\mathrm{P}_{\mathrm{D}}} \backslash \mathrm{P}_{\mathrm{d}} \rightarrow \mathrm{P}$ is completely continuous. Extend $\mathrm{T}$ (denote $\mathrm{T}$ yet) to $T: \overline{P_{D}} \rightarrow P$ which is completely continuous. Then, for $(x, y) \in \partial P_{d}$, we have

$$
d k t^{\gamma_{2}} \leqslant x(t), \quad y(t) \leqslant d, \quad t \in[0,1] .
$$

By Remark 2.8 and $\left(\mathrm{H}_{1}\right)-\left(\mathrm{H}_{3}\right)$, we get

$$
\mathrm{T}_{\mathfrak{i}}(x, y)(t) \geqslant\left(\frac{1}{4}\right)^{\gamma_{2}} k_{2} \int_{0}^{1} s(1-s)^{\gamma_{2}}\left(p_{i}(s) f_{i}\left(s, d k s^{\gamma_{2}}, d\right)+q_{i}(s) g_{i}\left(s, d, d k s^{\gamma_{2}}\right)\right) d s
$$




$$
\begin{aligned}
& \geqslant\left(\frac{1}{4}\right)^{\gamma_{2}} k_{2} \int_{0}^{1} s(1-s)^{\gamma_{2}}\left(p_{i}(s) f_{i}\left(s, d k s^{\gamma_{2}}, 1\right)+q_{i}(s) g_{i}\left(s, 1, d k s \gamma^{\gamma_{2}}\right)\right) d s \\
& \geqslant\left(\frac{1}{4}\right)^{\gamma_{2}} k_{2} \int_{0}^{1} s(1-s)^{\gamma_{2}}\left(d^{\lambda_{i}} k^{\lambda_{i}} p_{i}(s) f_{i}\left(s, s^{\gamma_{2}}, 1\right)+d^{\xi_{i}} k^{\eta_{i}} q_{i}(s) g_{i}\left(s, 1, s^{\gamma_{2}}\right)\right) d s \\
& \geqslant\left(\frac{1}{4}\right)^{\gamma_{2}} k_{2} d^{\max \left\{\lambda_{i}, \xi_{i}\right\}} k^{\max \left\{\lambda_{i}, \xi_{i}\right\}} \int_{0}^{1} s(1-s)^{\gamma_{2}}\left(p_{i}(s) f_{i}\left(s, s^{\gamma_{2}}, 1\right)+q_{i}(s) g_{i}\left(s, 1, s^{\gamma_{2}}\right)\right) d s \\
& \geqslant d=\|(x, y)\|_{1} \quad i=1,2, t \in\left[\frac{1}{4}, \frac{3}{4}\right] .
\end{aligned}
$$

This guarantees that

$$
\|T(x, y)\|_{1} \geqslant\|(x, y)\|_{1}, \quad \forall(x, y) \in \partial P_{d} .
$$

On the other hand, for any $(x, y) \in \partial P_{D}$, we have

$$
D k t^{\gamma_{2}} \leqslant x(t), \quad y(t) \leqslant D, \quad t \in[0,1] .
$$

Therefore, by Lemma 2.7, for any $(x, y) \in \partial P_{D}$ and $t \in[0,1]$, we have

$$
\begin{aligned}
\mathrm{T}_{i}(x, y)(t) \leqslant & k_{1} \int_{0}^{1} p_{1}(s) f_{1}\left(s, D, D k s^{\gamma_{2}}\right)+q_{1}(s) g_{1}\left(s, D k s{ }^{\gamma_{2}}, D\right) d s \\
& +k_{1} \int_{0}^{1} p_{2}(s) f_{2}\left(s, D, D k s^{\gamma_{2}}\right)+q_{2}(s) g_{2}\left(s, D k s{ }^{\gamma_{2}}, D\right) d s \\
\leqslant & k_{1} \int_{0}^{1} p_{1}(s) f_{1}\left(s, D, s^{\gamma_{2}}\right)+q_{1}(s) g_{1}\left(s, s^{\gamma_{2}}, D\right) d s \\
& +k_{1} \int_{0}^{1} p_{2}(s) f_{2}\left(s, D, s^{\gamma_{2}}\right)+q_{2}(s) g_{2}\left(s, s^{\gamma_{2}}, D\right) d s \\
\leqslant & k_{1} \int_{0}^{1} D^{\lambda_{1}} p_{1}(s) f_{1}\left(s, 1, s^{\gamma_{2}}\right)+D^{\xi_{1}} q_{1}(s) g_{1}\left(s, s^{\gamma_{2}}, 1\right) d s \\
& +k_{1} \int_{0}^{1} D^{\lambda_{2}} p_{2}(s) f_{2}\left(s, 1, s^{\gamma_{2}}\right)+D^{\xi_{2}} q_{2}(s) g_{2}\left(s, s^{\gamma_{2}}, 1\right) d s \\
\leqslant & k_{1} D^{\left.\max \lambda_{1}, \lambda_{2}, \xi_{1}, \xi_{2}\right\}}\left(\int_{0}^{1} p_{1}(s) f_{1}\left(s, 1, s^{\gamma_{2}}\right)+q_{1}(s) g_{1}\left(s, s^{\gamma_{2}}, 1\right) d s\right. \\
& \left.+\int_{0}^{1} p_{2}(s) f_{2}\left(s, 1, s^{\gamma_{2}}\right)+q_{2}(s) g_{2}\left(s, s^{\gamma_{2}}, 1\right) d s\right) \\
\leqslant & D=\|(x, y)\|_{1} .
\end{aligned}
$$

This guarantees that

$$
\|\mathrm{T}(x, y)\|_{1} \leqslant\|(x, y)\|_{1}, \quad \forall(x, y) \in \partial P_{D} .
$$

By the complete continuity of $\mathrm{T},(3.2)$ and (3.3), and Lemma 2.11, we obtain that $\mathrm{T}$ has a fixed point $\left(x^{*}, y^{*}\right)$ in $\overline{P_{D}} \backslash P_{d}$. Consequently, BVP (1.1) has a positive solution $\left(x^{*}, y^{*}\right)$ in $\overline{P_{D}} \backslash P_{d}$.

Next we prove that there exists a real number $0<m<1$ satisfying (3.1). Firstly, we show that for any $\theta \in\left(0, \frac{1}{2}\right)$ we have

$$
m t^{\gamma_{1}} \leqslant x^{*}(t) \leqslant \frac{1}{m} t^{\gamma_{1}}, \quad m t^{\gamma_{1}} \leqslant y^{*}(t) \leqslant \frac{1}{m} t^{\gamma_{1}}, \quad t \in[\theta, 1] .
$$

From Lemma 2.10, we know that $\left(x^{*}, y^{*}\right) \in P \backslash\{\theta\}$. So we obtain that

$$
0<k\left\|\left(x^{*}, y^{*}\right)\right\|_{1} t^{\gamma_{2}} \leqslant x^{*}(t), \quad y^{*}(t) \leqslant\left\|\left(x^{*}, y^{*}\right)\right\|_{1} .
$$

Let $h$ be a constant such that $\frac{\left\|\left(x^{*}, y^{*}\right)\right\|_{1}}{h}<1$ and $h>\frac{1}{d}>1$. By Lemma 2.5 we get 


$$
\begin{aligned}
x^{*}(t) \leqslant & k_{1} t^{\gamma_{1}}\left[\int_{0}^{1} p_{1}(s) f_{1}\left(s, h, \frac{k\left\|\left(x^{*}, y^{*}\right)\right\|_{1}}{h} s^{\gamma_{2}}\right)\right. \\
& +q_{1}(s) g_{1}\left(s, \frac{k\left\|\left(x^{*}, y^{*}\right)\right\|_{1}}{h} s^{\gamma_{2}}, h\right) d s \\
& +\int_{0}^{1} p_{2}(s) f_{2}\left(s, h, \frac{k\left\|\left(x^{*}, y^{*}\right)\right\|_{1}}{h} s^{\gamma_{2}}\right) \\
& \left.+q_{2}(s) g_{2}\left(s, \frac{k\left\|\left(x^{*}, y^{*}\right)\right\|_{1}}{h} s^{\gamma_{2}}, h\right) d s\right] \\
\leqslant & k_{1} t^{\gamma_{1}}\left[\int_{0}^{1} h^{\lambda_{1}+\mu_{1}}\left(k\left\|\left(x^{*}, y^{*}\right)\right\|_{1}\right)^{-\mu_{1}} p_{1}(s) f_{1}\left(s, 1, s^{\gamma_{2}}\right)\right. \\
& +h^{\xi_{1}+\eta_{1}}\left(k\left\|\left(x^{*}, y^{*}\right)\right\|_{1}\right)^{-\eta_{1}} q_{1}(s) g_{1}\left(s, s^{\gamma_{2}}, 1\right) d s \\
& +\int_{0}^{1} h^{\lambda_{2}+\mu_{2}}\left(k\left\|\left(x^{*}, y^{*}\right)\right\|_{1}\right)^{-\mu_{2}} p_{2}(s) f_{2}\left(s, 1, s^{\gamma_{2}}\right) \\
& \left.+h^{\xi_{2}+\eta_{2}}\left(k\left\|\left(x^{*}, y^{*}\right)\right\|_{1}\right)^{-\eta_{2}} q_{2}(s) g_{2}\left(s, s^{\gamma_{2}}, 1\right) d s\right] \\
= & C t^{\gamma_{1}}, \quad t \in[\theta, 1] .
\end{aligned}
$$

On the other hand, it is obvious to see that $\gamma_{2}-\gamma_{1} \geqslant 0$, where $\gamma_{1}=\min \{\alpha-1, \beta-1\}, \gamma_{2}=\max \{\alpha-1$, $\beta-1\}$. So we get

$$
\begin{aligned}
x^{*}(t) & \geqslant k\left\|\left(x^{*}, y^{*}\right)\right\|_{1} t^{\gamma_{2}} \\
& =k\left\|\left(x^{*}, y^{*}\right)\right\|_{1} t^{\gamma_{2}-\gamma_{1}} \gamma^{\gamma_{1}} \\
& \geqslant k\left\|\left(x^{*}, y^{*}\right)\right\|_{1} \theta^{\gamma_{2}-\gamma_{1}} t^{\gamma_{1}}, \quad t \in[\theta, 1] .
\end{aligned}
$$

In the same way, we can prove that $y^{*}(t) \leqslant C t^{\gamma_{1}}$ and $y^{*}(t) \geqslant k\left\|\left(x^{*}, y^{*}\right)\right\|_{1} \theta^{\gamma_{2}-\gamma_{1}} \gamma^{\gamma_{1}}, t \in[\theta, 1]$. Then, we pick out $m$ such that

$$
m=\min \left\{k \theta^{\left(\gamma_{2}-\gamma_{1}\right)}\left\|\left(x^{*}, y^{*}\right)\right\|_{1}, \frac{1}{C}, \frac{1}{2}\right\},
$$

which implies that (3.4) holds. Moreover, from the arbitrariness of $\theta$, we get that for any $t \in(0,1],(3.4)$ is satisfied. Specially, when $t=0$, by the boundary value conditions of $(1.1)$, we have $x^{*}(0)=y^{*}(0)=0$. So that we get that for any $t \in[0,1],(3.1)$ holds. This completes the proof of Theorem 3.1.

Theorem 3.2. Assume that conditions $\left(\mathrm{H}_{0}\right)-\left(\mathrm{H}_{3}\right)$ hold. If $\lambda_{i}+\mu_{i}<1$ and $\xi_{i}+\eta_{i}<1(i=1,2)$, then the BVP (1.1) has a unique positive solution $\left(x^{*}, y^{*}\right)$ and it satisfies (3.1).

Proof. By assuming the contrary, we find that the BVP (1.1) has a positive solution $\left(x_{*}, y_{*}\right)$ different from $\left(x^{*}, y^{*}\right)$. By (3.1) there exist $\rho_{1}, \rho_{2}>0$ such that

$$
\begin{aligned}
& \rho_{1} \mathrm{t}^{\gamma_{1}} \leqslant x^{*}(\mathrm{t}), \quad \mathrm{y}^{*}(\mathrm{t}) \leqslant \frac{1}{\rho_{1}} \mathrm{t}^{\gamma_{1}}, \quad \forall \mathrm{t} \in[0,1], \\
& \rho_{2} t^{\gamma_{1}} \leqslant x_{*}(t), \quad y_{*}(t) \leqslant \frac{1}{\rho_{2}} t^{\gamma_{1}}, \quad \forall t \in[0,1] .
\end{aligned}
$$

Hence, we have

$$
\begin{aligned}
& \rho_{1} \rho_{2} x_{*}(t) \leqslant x^{*}(t) \leqslant \frac{1}{\rho_{1} \rho_{2}} x_{*}(t), \\
& \rho_{1} \rho_{2} y_{*}(t) \leqslant y^{*}(t) \leqslant \frac{1}{\rho_{1} \rho_{2}} y_{*}(t), \quad \forall t \in[0,1] .
\end{aligned}
$$

Clearly, $\rho_{1} \rho_{2} \neq 1$. Put

$$
\rho^{*}=\sup \left\{\rho>0 \mid \rho x_{*}(t) \leqslant x^{*}(t) \leqslant \frac{1}{\rho} x_{*}(t), \rho y_{*}(t) \leqslant y^{*}(t) \leqslant \frac{1}{\rho} y_{*}(t), \forall t \in[0,1]\right\} .
$$


It is easy to see that $1>\rho^{*} \geqslant \rho_{1} \rho_{2}>0$ and

$$
\rho^{*} x_{*}(t) \leqslant x^{*}(t) \leqslant \frac{1}{\rho^{*}} x_{*}(t), \quad \rho^{*} y_{*}(t) \leqslant y^{*}(t) \leqslant \frac{1}{\rho^{*}} y_{*}(t), \quad \forall t \in[0,1] .
$$

By $\left(\mathrm{H}_{1}\right)$ and $\left(\mathrm{H}_{2}\right)$, we have

$$
\begin{aligned}
f_{i}\left(t, x^{*}(t), y^{*}(t)\right) & \geqslant f_{i}\left(t, \rho^{*} x_{*}(t), \frac{1}{\rho^{*}} y_{*}(t)\right) \\
& \geqslant\left(\rho^{*}\right)^{\lambda_{i}+\mu_{i}} f_{i}\left(t, x_{*}(t), y_{*}(t)\right) \\
& \geqslant\left(\rho^{*}\right)^{\sigma} f_{i}\left(t, x_{*}(t), y_{*}(t)\right), \\
g_{i}\left(t, x^{*}(t), y^{*}(t)\right) \geqslant & g_{i}\left(t, \rho^{*} x_{*}(t), \frac{1}{\rho^{*}} y_{*}(t)\right) \\
& \geqslant\left(\rho^{*}\right)^{\xi_{i}+\eta_{i}} g_{i}\left(t, x_{*}(t), y_{*}(t)\right) \\
& \geqslant\left(\rho^{*}\right)^{\sigma} g_{i}\left(t, x_{*}(t), y_{*}(t)\right), \quad i=1,2,
\end{aligned}
$$

where $\sigma=\max \left\{\lambda_{i}+\mu_{i}, \xi_{i}+\eta_{i}, i=1,2\right\}$ such that $\sigma<1$. Therefore, we have

$$
\begin{aligned}
x^{*}(t)= & T_{1}\left(x^{*}, y^{*}\right)(t)=\int_{0}^{1} G_{1}(t, s)\left[p_{1}(s) f_{1}\left(s, x^{*}(s), y^{*}(s)\right)+q_{1}(s) g_{1}\left(s, x^{*}(s), y^{*}(s)\right)\right] d s \\
& +\int_{0}^{1} H_{1}(t, s)\left[p_{2}(s) f_{2}\left(s, x^{*}(s), y^{*}(s)\right)+q_{2}(s) g_{2}\left(s, x^{*}(s), y^{*}(s)\right)\right] d s \\
\geqslant & \left(\rho^{*}\right)^{\sigma}\left[\int_{0}^{1} G_{1}(t, s)\left[p_{1}(s) f_{1}\left(s, x^{*}(s), y^{*}(s)\right)+q_{1}(s) g_{1}\left(s, x^{*}(s), y^{*}(s)\right)\right] d s\right. \\
& \left.+\int_{0}^{1} H_{1}(t, s)\left[p_{2}(s) f_{2}\left(s, x^{*}(s), y^{*}(s)\right)+q_{2}(s) g_{2}\left(s, x^{*}(s), y^{*}(s)\right)\right] d s\right] \\
= & \left(\rho^{*}\right)^{\sigma} T_{1}\left(x_{*}, y_{*}\right)(t)=\left(\rho^{*}\right)^{\sigma} x_{*}(t) .
\end{aligned}
$$

Similarly, we can get

$$
y^{*}(t) \geqslant\left(\rho^{*}\right)^{\sigma} y_{*}(t), \quad x_{*}(t) \geqslant\left(\rho^{*}\right)^{\sigma} x^{*}(t), \quad y_{*}(t) \geqslant\left(\rho^{*}\right)^{\sigma} y^{*}(t) .
$$

Noticing that $\left(\rho^{*}\right)^{\sigma}>\rho^{*}\left(0<\rho^{*}, \sigma<1\right)$, we get to a contradiction with the maximality of $\rho^{*}$. Thus, the BVP (1.1) has a unique positive solution $\left(x^{*}, y^{*}\right)$. This completes the proof of Theorem 3.2.

Remark 3.3. Compared with the result in $[3,21]$, we can see that for any $\alpha \in(n-1, n], \beta \in(m-$ $1, m], n, m \in \mathbb{N}$, we can get the uniqueness of positive solutions of the BVP (1.1). That is, we do not need the condition of $\alpha=\beta$. So our result is better than that in [3,21].

\section{An example}

We give an explicit example to illustrate our main result in Section 3. Let us consider the singular differential system with coupled boundary conditions

$$
\left\{\begin{array}{l}
D_{0^{+}}^{\frac{5}{2}} x(t)+\frac{\sqrt{x}}{\sqrt[3]{y(1-t) t}}+\frac{\sqrt[3]{y}}{\sqrt{x}}=0, \quad t \in(0,1), \\
D_{0^{+}}^{\frac{7}{2}} y(t)+\frac{\sqrt[3]{x}}{\sqrt{y}}+\frac{\sqrt{y}}{\sqrt[3]{x(1-t) t}}=0, \quad t \in(0,1), \\
x(0)=x^{\prime}(0)=0, \quad x(1)=y\left(\frac{1}{3}\right)+y\left(\frac{1}{2}\right), \\
y(0)=y^{\prime}(0)=y^{\prime \prime}(0)=0, \quad y(1)=\int_{0}^{1} x(s) d s^{2} .
\end{array}\right.
$$


Let $\alpha=\frac{5}{2}, \beta=\frac{7}{2}$,

$$
\begin{aligned}
& f_{1}(t, x, y)=\frac{\sqrt{x}}{\sqrt[3]{y(1-t) t}}, \quad g_{1}(t, x, y)=\frac{\sqrt[3]{y}}{\sqrt{x}} \\
& f_{2}(t, x, y)=\frac{\sqrt[3]{x}}{\sqrt{y}}, \quad g_{2}(t, x, y)=\frac{\sqrt{y}}{\sqrt[3]{x(1-t) t}} \\
& \mathrm{a}(\mathrm{t})=\mathrm{b}(\mathrm{t})=1, \\
& A(t)= \begin{cases}0, & t \in\left[0, \frac{1}{3}\right), \\
1, & t \in\left[\frac{1}{3}, \frac{1}{2}\right), \\
2, & t \in\left[\frac{1}{2}, 1\right],\end{cases} \\
& B(t)=t^{2} . \\
& \lambda_{1}=\mu_{2}=\frac{1}{2}, \quad \lambda_{2}=\mu_{1}=\frac{1}{3}, \quad \xi_{1}=\eta_{2}=\frac{1}{2}, \quad \xi_{2}=\eta_{1}=\frac{1}{3},
\end{aligned}
$$

then

$$
\begin{aligned}
& \int_{0}^{1} p_{1} f_{1}(s, 1,1-s) d s=B\left(\frac{2}{3}, \frac{1}{6}\right), \quad \int_{0}^{1} q_{1} g_{1}(s, 1-s, 1) d s=B\left(1, \frac{1}{2}\right), \\
& \int_{0}^{1} p_{2} f_{2}(s, 1,1-s) d s=B\left(1, \frac{1}{2}\right), \int_{0}^{1} q_{2} g_{2}(s, 1-s, 1) d s=B\left(\frac{2}{3}, \frac{1}{3}\right) .
\end{aligned}
$$

So all conditions of Theorems 3.1 and 3.2 are satisfied for (4.1) and our conclusion follows from Theorems 3.1 and 3.2 , namely the BVP (4.1) has a unique positive solution $\left(x^{*}, y^{*}\right)$ and there exists a real number $0<\mathrm{m}<1$ such that

$$
m t^{\gamma_{1}} \leqslant x^{*}(t) \leqslant \frac{1}{m} t^{\gamma_{1}}, \quad m t^{\gamma_{1}} \leqslant y^{*}(t) \leqslant \frac{1}{m} t^{\gamma_{1}}, \quad t \in[0,1],
$$

where $\gamma_{1}=\min \{\alpha-1, \beta-1\}=\frac{3}{2}$.

\section{Conclusions}

In this paper, by using the mixed monotone operators and the Guo-Krasnosel'skii fixed point theorem, we have established the existence and uniqueness of positive solutions for a class of singular fractional differential systems with coupled integral boundary conditions for any real number $\alpha, \beta \in(0,+\infty)$. It is worth noting that in this paper we divide the functions into the former of $f_{i}+g_{i}(i=1,2)$ and add different conditions to $f_{i}$ and $g_{i}$. From this point, our result is more general than that in $[3,21]$.

\section{Acknowledgment}

The authors were supported financially by the National Natural Science Foundation of China (11371221, 11571296).

\section{References}

[1] B. Ahmad, J. J. Nieto, Existence results for a coupled system of nonlinear fractional differential equations with three-point boundary conditions, Comput. Math. Appl., 58 (2009), 1838-1843. 1

[2] C.-Z. Bai, J.-X. Fang, The existence of a positive solution for a singular coupled system of nonlinear fractional differential equations, Appl. Math. Comput., 150 (2004), 611-621. 1

[3] Y.-J. Cui, L.-S. Liu, X.-U. Zhang, Uniqueness and existence of positive solutions for singular differential systems with coupled integral boundary value problems, Abstr. Appl. Anal., 2013 (2013), 9 pages. 1, 3.3, 5 
[4] W.-Q. Feng, S.-R. Sun, Z.-L. Han, Y.-G. Zhao, Existence of solutions for a singular system of nonlinear fractional differential equations, Comput. Math. Appl., 62 (2011), 1370-1378. 1

[5] D.-J. Guo, V. Lakshmikantham, Nonlinear problems in abstract cones, Notes and Reports in Mathematics in Science and Engineering, Academic Press, Inc., Boston, MA, (1988). 2.11

[6] L.-M. Guo, L.-S. Liu, Y.-H. Wu, Uniqueness of iterative positive solutions for the singular fractional differential equations with integral boundary conditions, Bound. Value Probl., 2016 (2016), 20 pages. 1

[7] J. Henderson, R. Luca, Existence and multiplicity for positive solutions of a system of higher-order multi-point boundary value problems, NoDEA Nonlinear Differential Equations Appl., 20 (2013), 1035-1054. 1

[8] J. Henderson, R. Luca, Existence and multiplicity of positive solutions for a system of fractional boundary value problems, Bound. Value Probl., 2014 (2014), 17 pages. 1

[9] J. J. Jiang, L.-S. Liu, Existence of solutions for a sequential fractional differential system with coupled boundary conditions, Bound. Value Probl., 2016 (2016), 15 pages. 1

[10] A. A. Kilbas, H. M. Srivastava, J. J. Trujillo, Theory and applications of fractional differential equations, North-Holland Mathematics Studies, Elsevier Science B.V., Amsterdam, (2006). 2, 2.3, 2.4

[11] W.-W. Liu, L.-S. Liu, Y.-H. Wu, Positive solutions of a singular boundary value problem for systems of second-order differential equations, Appl. Math. Comput., 208 (2009), 511-519. 1

[12] L. Liu, F. Sun, X. Zhang, Y. Wu, Bifurcation analysis for a singular differential system with two parameters via to degree theory, Nonlinear Anal. MC, 22 (2017), 31-50.

[13] S.-Y. Liu, G.-T. Wang, L.-H. Zhang, Existence results for a coupled system of nonlinear neutral fractional differential equations, Appl. Math. Lett., 26 (2013), 1120-1124. 1

[14] A. Lomtatidze, L. Malaguti, On a nonlocal boundary value problem for second order nonlinear singular differential equations, Georgian Math. J., 7 (2000), 133-154. 2

[15] I. Podlubny, Fractional differential equations, An introduction to fractional derivatives, fractional differential equations, to methods of their solution and some of their applications, Mathematics in Science and Engineering, Academic Press, Inc., San Diego, CA, (1999). 2

[16] H. A. H. Salem, On the existence of continuous solutions for a singular system of non-linear fractional differential equations, Appl. Math. Comput., 198 (2008), 445-452. 1

[17] S. G. Samko, A. A. Kilbas, O. I. Marichev, Fractional integrals and derivatives, Theory and applications, Edited and with a foreword by S. M. Nikolskiǔ, Translated from the 1987 Russian original, Revised by the authors, Gordon and Breach Science Publishers, Yverdon, (1993). 2

[18] X.-W. Su, Boundary value problem for a coupled system of nonlinear fractional differential equations, Appl. Math. Lett., 22 (2009), 64-69. 1

[19] F.-L. Sun, L.-S. Liu, X.-U. Zhang, Y.-H. Wu, Spectral analysis for a singular differential system with integral boundary conditions, Mediterr. J. Math., 13 (2016), 4763-4782.

[20] M. ur Rehman, R. Ali Khan, A note on boundary value problems for a coupled system of fractional differential equations, Comput. Math. Appl., 61 (2011), 2630-2637.

[21] Y. Wang, L.-S. Liu, X.-U. Zhang, Y.-H. Wu, Positive solutions for ( $\mathrm{n}-1,1)$-type singular fractional differential system with coupled integral boundary conditions, Abstr. Appl. Anal., 2014 (2014), 14 pages. 1, 3.3, 5

[22] Y. Wang, L.-S. Liu, X.-U. Zhang, Y.-H. Wu, Positive solutions of an abstract fractional semipositone differential system model for bioprocesses of HIV infection, Appl. Math. Comput., 258 (2015), 312-324.

[23] W.-G. Yang, Positive solutions for a coupled system of nonlinear fractional differential equations with integral boundary conditions, Comput. Math. Appl., 63 (2012), 288-297. 1

[24] C.-J. Yuan, Multiple positive solutions for $(\mathrm{n}-1,1)$-type semipositone conjugate boundary value problems of nonlinear fractional differential equations, Electron. J. Qual. Theory Differ. Equ., 2010 (2010), 12 pages. 2.6

[25] C.-J. Yuan, Two positive solutions for $(n-1,1)$-type semipositone integral boundary value problems for coupled systems of nonlinear fractional differential equations, Commun. Nonlinear Sci. Numer. Simul., 17 (2012), 930-942. 1 